\title{
A 3D IPSC-differentiation model identifies interleukin-3 as a regulator of early human hematopoletic specification
}

Haematologica 2021

Volume 106(5):1354-1367

\section{Correspondence:}

NICO LACHMANN

lachmann.nico@mh-hannover.de

Received: May 28, 2019.

Accepted: March 25, 2020.

Pre-published: April 23, 2020.

https://doi.org/10.3324/haematol.2019.228064

(C)2021 Ferrata Storti Foundation

Material published in Haematologica is covered by copyright. All rights are reserved to the Ferrata Storti Foundation. Use of published material is allowed under the following terms and conditions:

https://creativecommons.org/licenses/by-nc/4.0/legalcode. Copies of published material are allowed for personal or internal use. Sharing published material for non-commercial purposes is subject to the following conditions:

https://creativecommons.org/licenses/by-nc/4.0/legalcode, sect. 3. Reproducing and sharing published material for commercial purposes is not allowed without permission in writing from the publisher.

\author{
Mania Ackermann, ${ }^{1,2}$ Kathrin Haake, ${ }^{1,2}$ Henning Kempf, ${ }^{2,3,4}$ Paul Kaschutnig, ${ }^{5}$ \\ Anna-Carina Weiss, ${ }^{6}$ Ariane H.H. Nguyen, ${ }^{1,2}$ Markus Abeln, ${ }^{7}$ Sylvia Merkert, ${ }^{2,3}$ \\ Mark Phillip Kühnel, ${ }^{8,9}$ Dorothee Hartmann, ${ }^{10}$ Danny Jonigk, ${ }^{2,8,9}$ \\ Thomas Thum, ${ }^{2,10}$ Andreas Kispert, ${ }^{6}$ Michael D. Milsom ${ }^{5}$ \\ and Nico Lachmann ${ }^{1,2}$
}

\begin{abstract}
${ }^{1}$ Institute of Experimental Hematology, Hannover Medical School, Hannover, Germany; ${ }^{2}$ REBIRTH Center for Translational Regenerative Medicine, Hannover Medical School, Hannover, Germany; ${ }^{3}$ Leibniz Research Laboratories for Biotechnology and Artificial Organs (LEBAO), Department of Cardiothoracic, Transplantation and Vascular Surgery, Hannover Medical School, Hannover, Germany; ${ }^{4}$ Department of Stem Cell Discovery, Novo Nordisk A/S, Maaloev, Denmark; ${ }^{5}$ German Cancer Research Center (DKFZ), Division of Experimental Hematology and Heidelberg Institute for Stem Cell Technology (HI-STEM), Heidelberg, Germany; ${ }^{6}$ Institute of Molecular Biology, Hannover Medical School, Hannover, Germany; ${ }^{7}$ Institute of Clinical Biochemistry, Hannover Medical School, Hannover, Germany; ${ }^{8}$ Institute for Pathology, Hannover Medical School, Hannover, Germany; ${ }^{9}$ Biomedical Research in Endstage and Obstructive Lung Disease (BREATH), German Center for Lung Research, Hannover, Germany and ${ }^{10}$ Institute of Molecular and Translational Therapeutic Strategies (IMTTS), Hannover Medical School, Hannover, Germany
\end{abstract}

\section{ABSTRACT}

H ematopoietic development is spatiotemporally tightly regulated by defined cell-intrinsic and extrinsic modifiers. The role of cytokines has been intensively studied in adult hematopoiesis; however, their role in embryonic hematopoietic specification remains largely unexplored. Here, we used induced pluripotent stem cell (iPSC) technology and established a 3-dimensional (3D), organoid-like differentiation system ("hemanoid") maintaining the structural cellular integrity to evaluate the effect of cytokines on embryonic hematopoietic development. We show that defined stages of early human hematopoietic development were recapitulated within the generated hemanoids. We identified $\mathrm{KDR}^{+} / \mathrm{CD} 34^{\text {high }} / \mathrm{CD} 144^{+} / \mathrm{CD} 43^{-} / \mathrm{CD}^{2} 5^{-}$hemato-endothelial progenitors (HEP) forming organized, vasculature-like structures and giving rise to $\mathrm{CD} 34^{\mathrm{low}} / \mathrm{CD} 144^{-} / \mathrm{CD} 43^{+} / \mathrm{CD} 45^{+}$hematopoietic progenitor cells. We demonstrate that the endothelial to hematopoietic transition of HEP is dependent on the presence of interleukin 3 (IL-3). Inhibition of IL-3 signaling blocked hematopoietic differentiation and arrested the cells in the HEP stage. Thus, our data suggest an important role for IL-3 in early human hematopoiesis by supporting the endothelial to hematopoietic transition of HEP and highlight the potential of a hemanoid-based model to study human hematopoietic development.

\section{Introduction}

Different cell-intrinsic and extrinsic factors direct the specification of early mesodermal progenitor cells towards hematopoietic stem cells (HSC), which arise in the aorta-gonad-mesonephros (AGM) region, mature in the fetal liver and finally home and reside in the bone marrow. This process is spatiotemporally tightly regulated and proceeds through two distinct stages: a primitive and a definitive hematopoietic program. ${ }^{1}$ While embryonic macrophages and nucleated red blood cells are generated early in development in the first wave of primitive hematopoiesis, lymphoid cells, definitive erythro-myeloid progenitors and long-term repopulating 
HSC are only generated at later stages by the definitive hematopoietic program. ${ }^{2}$ In several studies, the hemogenic endothelium (HE), which is predominantly present in the AGM, has been identified as the primary cell source responsible for the emergence of definitive hematopoietic cells. ${ }^{3-5}$ The emergence of hematopoietic cells from the HE (also referred to as endothelial to hematopoietic transition [EHT]), is controlled by distinct cell-intrinsic signals mediated by transcription factor (TF) activation, as well as important cell-extrinsic factors such as cell-cell interactions, extracellular matrix molecules, and cytokines. ${ }^{6}$

The latter are of great interest as diverse cytokines have been shown to regulate the differentiation of mature blood cells from adult HSC. ${ }^{7}$ Among others, interleukin-3 (IL-3), formerly known as multi colony stimulating factor, has been implicated in survival, proliferation, and differentiation of HSC and hematopoietic progenitor populations. ${ }^{8}$ Although disruption of $I L-3$ or IL $3 \mathrm{ra} / \mathrm{b}$ genes is not lethal in mice, ${ }^{9,10} \mathrm{IL}-3$ has been shown to induce amplification of the HSC pool in the midgestation mouse embryo. ${ }^{11}$ The same study suggests that the mechanism by which IL-3 expression facilitates the detection of HSC in early embryonic day (E) E10 AGM and yolk sac (YS) is mediated by supporting (i) either the survival and/or proliferation of a limiting number of previously undetectable HSC or (ii) even the emergence of new HSC. Along the same lines, IL-3 has been shown to positively regulate hemangioblast development in the murine AGM, by stimulating the differentiation and proliferation of fetal liver kinase (Flk) $1^{+}$mesodermal progenitors. ${ }^{12}$

While most of these findings are derived from non-vertebrate or murine studies, little is known about the different cell-intrinsic and extrinsic regulators of human embryonic hematopoietic development. Similarly, the role of IL-3 in human hematopoietic specification has remained elusive. Given the difficulties to study these processes in humans, in vitro hematopoietic differentiation of human pluripotent stem cells (hPSC) provides an invaluable platform to study hematopoietic specification and identify factors critically regulating the human EHT. ${ }^{13,14}$ In vitro hPSC-derived hematopoietic development has been shown to proceed through distinct stages including $\mathrm{KDR}^{+}$ mesodermal progenitors, hemogenic endothelium and finally the emergence of hematopoietic stem/progenitor cells. Whereas the two waves of primitive and definitive hematopoietic development are spatiotemporally regulated in vivo, ${ }^{15}$ hPSC-derived hematopoiesis often yields a mixture of cells derived from the primitive and definitive hematopoietic program. ${ }^{6,16}$ Several modulators, such as the Activin/Nodal- and WNT-signaling pathways, have been identified to be important for the emergence of definitive hematopoietic precursor cells. ${ }^{17}$ However, even when considering the most advanced in vitro differentiation protocols available to date, only very limited hematopoietic engraftment and a restriction to the myeloid differentiation fate have been observed for hPSC-derived HSC, ${ }^{6}$ highlighting the necessity to better characterize PSCderived hematopoiesis.

One factor often neglected in PSC-based hematopoietic differentiation systems is the influence of the microenvironment. The development of HSC in the AGM is critically regulated by microenvironmental signals from the surrounding mesenchyme, hematopoietic and endothelial cells. ${ }^{18}$ In order to gain further insights into the cues regulating early developmental processes, different organoid systems have been introduced and shown to closely mimic features of e.g., lung, intestine or brain development. ${ }^{19}$ Organoid-based hematopoietic differentiation systems providing a 3-dimensional (3D) niche for developing HSC may also be of high relevance to gain novel insights into transcriptional regulators of human hematopoietic development.

Considering the important role of IL-3 in early murine hematopoietic development, we sought to evaluate the regulatory role of IL-3 in early human hematopoiesis. Using an organoid-like hematopoietic differentiation protocol, we demonstrate that the addition of IL-3 alone is sufficient to induce the continuous production of immature, myeloid progenitor cells. We furthermore provide evidence that EHT is IL-3 dependent, highlighting a very early effect of this cytokine on hematopoietic specification of hPSC. Thus, we have developed an organoid-like hematopoietic differentiation system for hPSC and unravel the IL-3 signaling cascade as an important regulator of early human hematopoietic specification.

\section{Methods}

\section{Human induced pluripotent stem cell cultivation}

hPSC were cultured on irradiated murine embryonic fibroblasts (MEF) derived from CF1 mice in induced PSC (iPSC)-medium (knockout DMEM, 20\% knockout serum replacement, $1 \mathrm{mM} \mathrm{L}$ glutamine, 1\% NEAA, 1\% penicillin/streptomycin [all Invitrogen], $0.1 \mathrm{mM} \beta$-mercaptoethanol [Sigma-Aldrich], and $10 \mathrm{ng} / \mathrm{mL}$ basic fibroblast growth factor [bFGF] [Peprotech] $).{ }^{20}$ Cells were passaged every 7-10 days using incubation with collagenase IV.

\section{Human induced pluripotent stem cell differentiation}

Hematopoietic differentiation was performed following our establish protocol. ${ }^{21}$ In brief, embryoid bodies (EB) were generated from iPSC colonies disrupted with collagenase by cultivation of the fragments on an orbital shaker ( $85 \mathrm{rpm}$ ) in iPSC-medium without bFGF but supplemented with $10 \mu \mathrm{M}$ Rock inhibitor (Y-27632; Tocris). Medium was changed after 2-3 days. After 5 days, EB were manually selected and transferred to adherent plates in differentiation medium I (APEL [II] medium, Stem Cell Technologies or prepared according to a previously published protocol ${ }^{22}$ ) supplemented with $50 \mathrm{ng} / \mathrm{mL}$ IL-3, a combination of $25 \mathrm{ng} / \mathrm{mL})^{22}$ IL-3 and $50 \mathrm{ng} / \mathrm{mL}$ M-CSF, $250 \mathrm{ng} / \mathrm{mL}$ of a rat anti-human IL-3 antibody (Clone BVD8-3G11, BD Bioscience) or without cytokine addition. Alternatively, the last step of differentiation was carried out in suspension as described recently. ${ }^{23}$ Floating hematopoietic cells were harvested from the medium and filtered through a 150$\mu \mathrm{m}$ mesh from day 14 onwards while hemanoids remained in culture.

For terminal differentiation, cells harvested from the hemanoids were cultured in differentiation medium II (RPMI 1640 medium supplemented with 10\% fetal serum, $2 \mathrm{mM}$ L-glutamine, $1 \%$ penicillin-streptomycin, all from Invitrogen) supplemented with $100 \mathrm{ng} / \mathrm{mL}$ human macrophage (hM) colony stimulating factor (CSF) (hM-CSF), human granulocyte-CSF (hG-CSF), or human macrophage-granuclocyte-CSF (hGM-CSF), for 7-10 days.

\section{Whole transcriptome analysis}

Gene expression analyses were performed using Illumina HumanHT12v4 BeadChips ${ }^{\circledR}$ at the Genomics and Proteomics Core facility of the DKFZ-Heidelberg. Gene expression data were quantile-normalized and subsequently anlayzed using $\mathrm{R} /$ Bioconductor through the graphical user interface Chipster 
(v3.8, chipstercsc.fi). Differentially regulated genes were called using two group tests (empirical Bayes). Differential expression thresholds were set to $\log _{2}$-fold change $\geq 1$ (upregulated) or $\leq 1$ (downregulated) with an adjusted $P$-value of $\leq 0.05$ (corrected with Benjamini Hochberg equasion).

Heatmap clustering analysis and generation of GO-based heatmaps were performed in Omics Explorer (version 3.4). Gene ontology analysis of biological processes, molecular function and cell type classification based on The Human Gene Atlas were conducted using Enrichr (https://amp.pharm.mssm.edu/Enrichr). Gene set enrichment analysis (GSEA) was performed using GSEA v3.0, Broad Institute.

\section{Culture of OP9 stromal cells}

Transgenic OP9 cells expressing Delta-like ligand (DLL) 1 or DLL4 were cultured in $\alpha$-MEM supplemented with $20 \%$ fetal calf

A

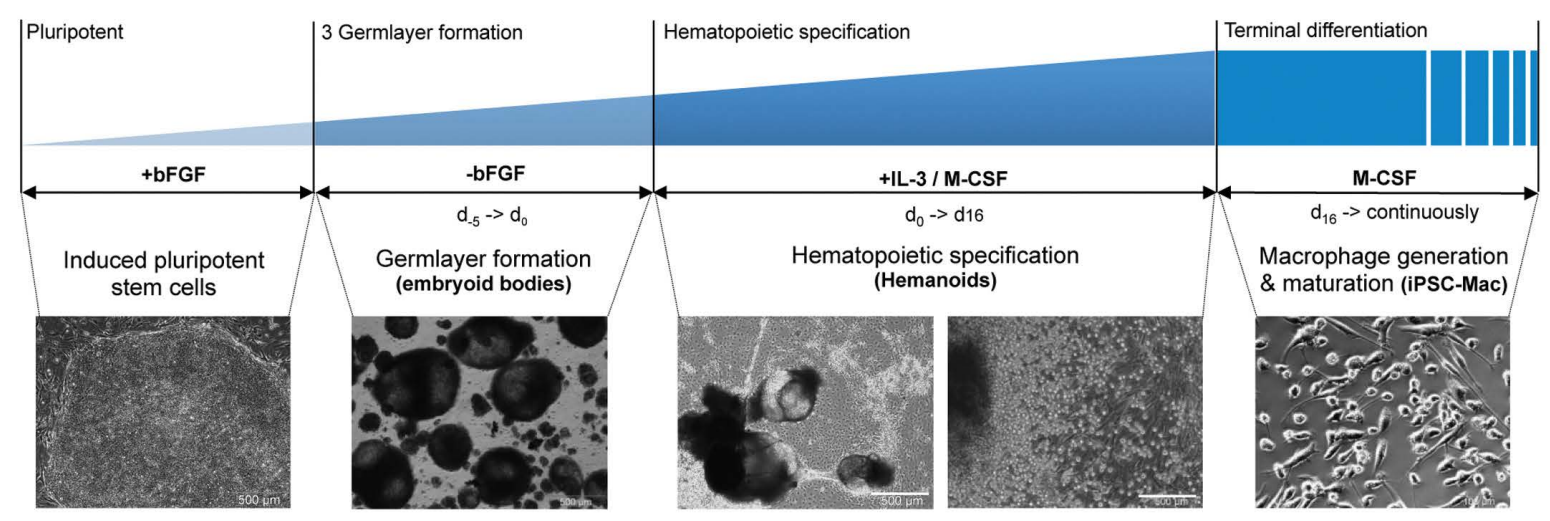

B

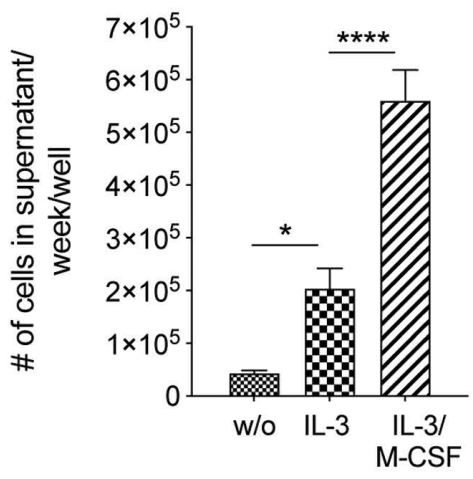

C

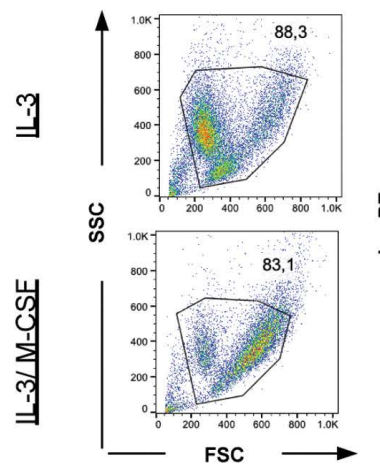

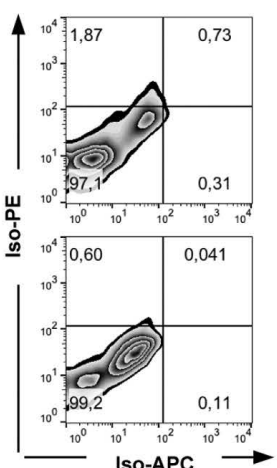

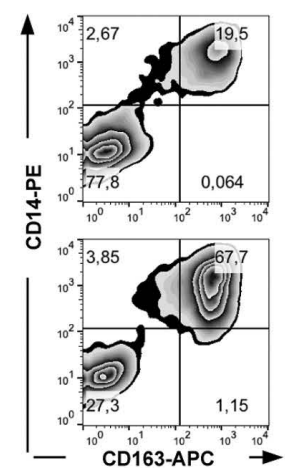

D

W IL3 EA IL3\&M-CSF

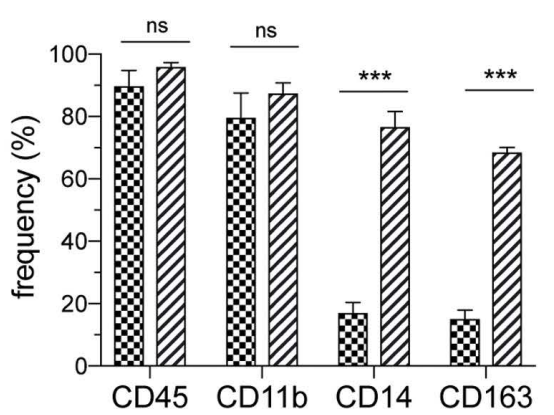

E

E CFU-GM

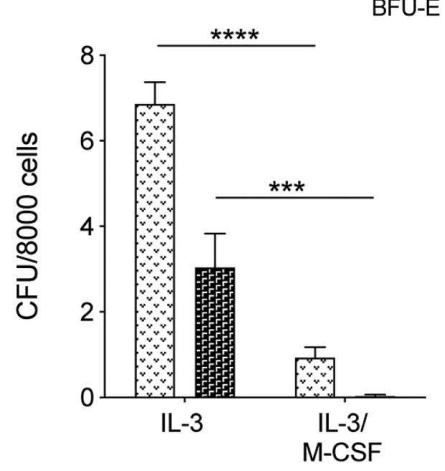

$\mathrm{F}$
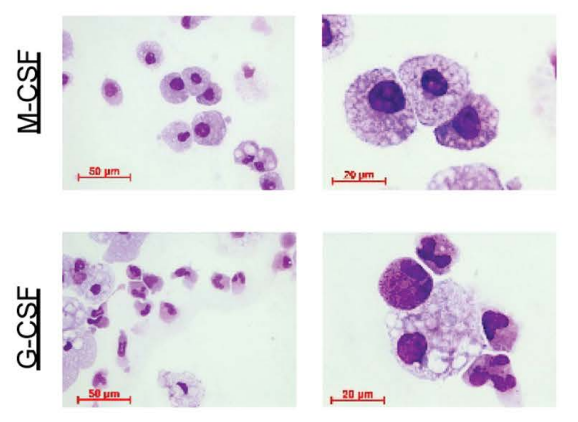

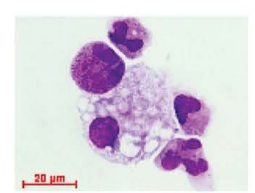

Figure 1. Effect of IL-3 on hematopoietic differentiation of human induced pluripotent stem cells. (A) Schematic representation and brightfield microscopy of different stages within the employed hematopoietic differentiation protocol. From left to right: pluripotent stem cells, embryoid bodies, hemanoids (scale bars: 500 um) and induced pluripotent stem cell (iPSC)-derived macrophages (scale bar: $100 \mu \mathrm{m}$ ). (B) Quantity of total cells harvested from the supernatant of the hemanoids cultured in different differentiation conditions (without [W/o] cytokines, with only IL-3 or with IL-3/M-CSF) per week and well ( $n=9-10$, mean \pm standard error of the mean [SEM]). (C and D) Analysis of cells harvested from differentiation cultures with only IL-3 and IL-3/M-CSF. (C) Representative flow cytometry analysis of specific surface marker expression (percentage of gated population is indicated) and (D) quantification of $n=3$, mean $\pm S E M$. (E) Colony forming units (CFU) granulocyte/monocyte (GM) and CFU-granulocyte/erythrocyte/monocyte/megakaryocyte (GEMM) or erythroid burst forming unites (BFU-E) of cells harvested from different culture conditions $(n=3-4$, mean \pm SEM). (F) Cytospin staining of cells harvested from cultures with only IL-3 that were further matured for 1 week in medium with M-CSF (upper row) or G-CSF (lower row), respectively (scale bar: $50 \mu \mathrm{m}$ and $20 \mu \mathrm{m}$, respectively). $* P<0.05, * * P<0.01, * * * P<0.001, * * * * P<0.0001$; statistical significance was assessed using (B) one-way ANOVA with Tukey's multiple comparison test and (D and E) two-way ANOVA with Sidak's multiple comparisons test. 
serum (FCS) (Hyclone), 1 mM L-glutamine, 1\% NEAA, 1\% penicillin/streptomycin (all from Invitrogen) and $0,2 \% \quad \beta$-mercaptoethanol (Sigma-Aldrich). In order to counteract transgene silencing and maintain a high expression of DLL, cells were cultured with $2 \mu \mathrm{g} / \mathrm{mL}$ puromycin (Roth). Cells were passaged twice a week using trypsin. For EHT assay, OP9 DLL1 and DLL4 cells were mixed 50:50 and seeded in 12-well plates (TPP, Switzerland).

\section{Endothelial to hematopoietic transition culture}

In order to facilitate endothelial to hematopoietic transition outside the hemanoids, fluorescence-activated cell sorting (FACS)- purified HEP $\left(\mathrm{CD} 34^{+} / \mathrm{CD} 144^{+} / \mathrm{CD} 45^{-}\right)$were cultured on overgrown OP9 stromal cells expressing DLL1 and DLL4 in differentiation medium I supplemented with $50 \mathrm{ng} / \mathrm{mL}$ IL-3 (or as a control in the absence of any cytokines). Cells were analyzed by microscopy or flow cytometry 7-14 days post sorting.

\section{Ethical approval}

Generation and use of hiPsc has been approved by the local ethical committee of Hannover Medical School (Approval No.: 21272014 and 7870_BO_K_2018). Human CD34 cells were isolated following written informed consent of the donor, which has been
A

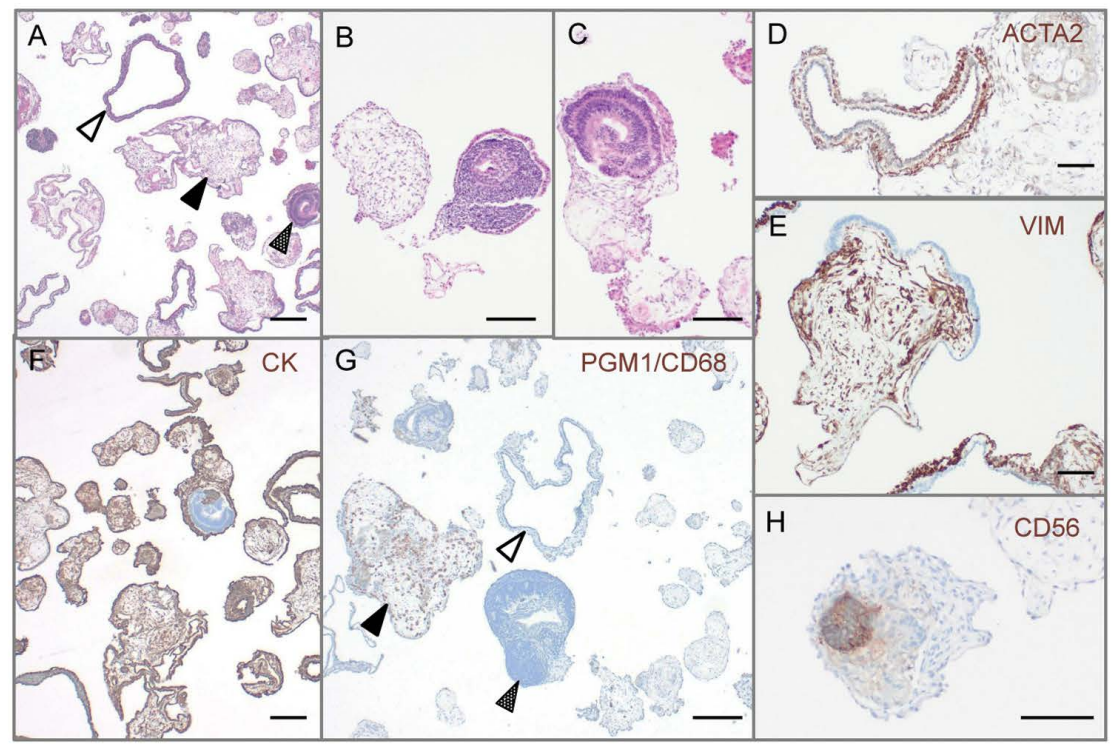

B

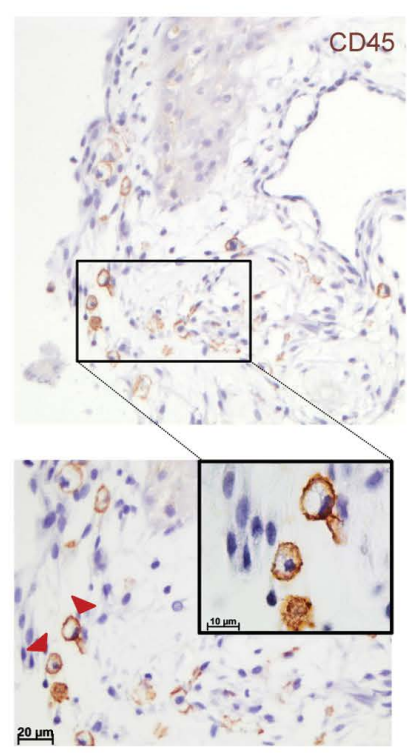

C

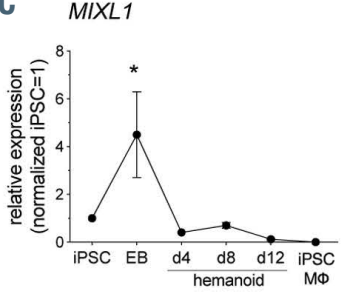

$K D R 1$
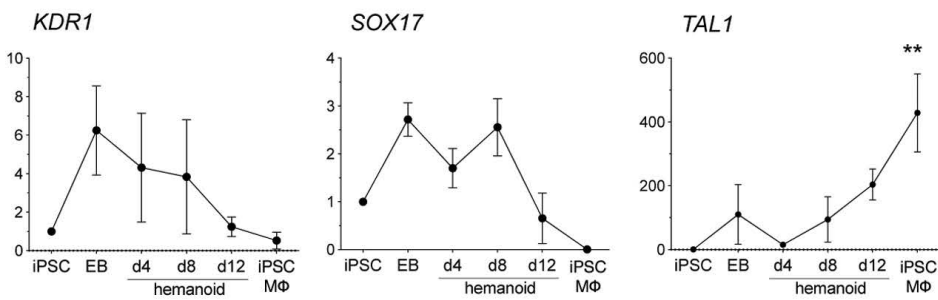

D
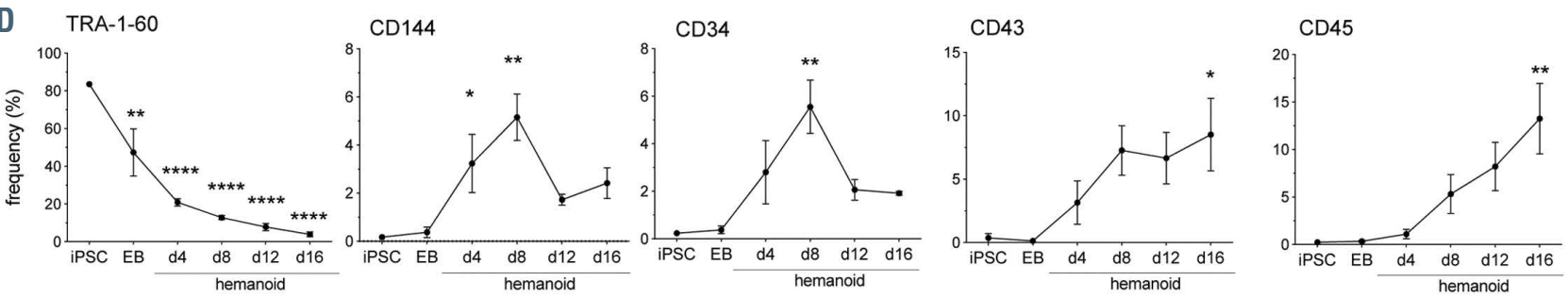

Figure 2. Characterization of hemanoids during hematopioietic specification. (A) Analysis of paraffin sections of hemanoids day 16. A: glandular structure (white arrow), immature mesodermal (black arrow) and neuroectodermal structures (spotted arrow), B: mesodermal structures of varying degrees of differentiation, C: neuroectodermal formation (CD56 positive [H]), D: smooth muscle actin (ATCA2) positive myoepithelial cells in glandular structures, E: vimentin (VIM) positive mesodermal cells, F: pan cytokeratin (CK) positive epithelial structures with varying degrees of positivity, G: PGM1/CD68 mononuclear MO precursors (black arrow) with immature mesoderm and glandular structures (white and spotted arrowheads respectively), H: CD56 positive neuroectodermal formation. Scale bar represents $100 \mu \mathrm{m})$. (B) Immunohistochemical analyses of CD45 expression in hemanoids day 16 (bottom panel: arrowheads indicate CD45 positive cells in the magnified area). (C) Analysis of transcription factor expression at defined stages of differentiation (induced pluripotent stem cell [iPSC], embryoid bodies [EB], hemanoids day $4,8$ and 12 and iPSC-derived macrophages [iPSC-M $\Phi]$ ]) by quantitative real time polymerase chain reaction (qRT-PCR) ( $\mathrm{n}=3$, mean \pm Standard error of the mean [SEM]). (D) Analysis of surface marker expression at defined stages of differentiation (iPSC, EB, hemanoids day $4,8,12$ and 16$)$ by flow cytometry ( $n=3$, mean $\pm S E M$ ). $\star P<0.05, * * P<0.01, * * * P<0.001, * * * * P<0.0001$ statistical significance was assessed using one-way ANOVA with Dunnetts multiple comparison test. 
approved by the local ethical committee of Hannover Medical School (No.: 2127-2014 and 1303-2012).

\section{Results}

\section{Interleukin 3 allows the generation of immature myeloid cells from induced pluripotent stem cell-derived hemanoids}

We recently established a hematopoietic differentiation system that facilitates the generation of different mature myeloid cells from human iPSC via the generation of $3 \mathrm{D}$ complexes, which we refer to as "hemanoids" (Figure 1A).
These complexes are, depending on the cytokine administration, able to produce different myeloid cell types such as macrophages and granulocytes ${ }^{13}$ or erythrocytes ${ }^{24}$ for several months, suggesting the development and maintenance of a hematopoietic progenitor population. While we have intensively employed this approach for disease modeling studies and cell-based therapies, ${ }^{25-29}$ we here sought to utilize these organoid-like $3 \mathrm{D}$ complexes to study human embryonic hematopoietic development in vitro.

In order to elucidate the effect of IL-3 on the hematopoietic differentiation of hiPSC, we first evaluated the effect of cytokine withdrawal in our hemanoid-based differenti-
A

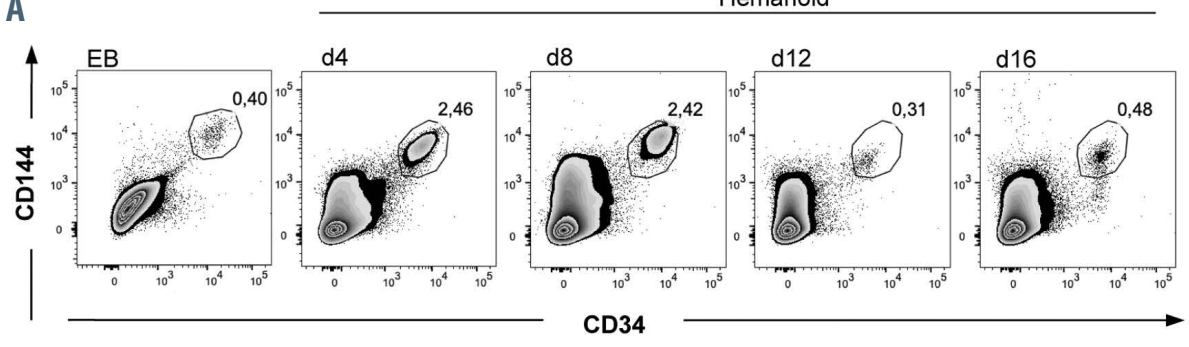

B

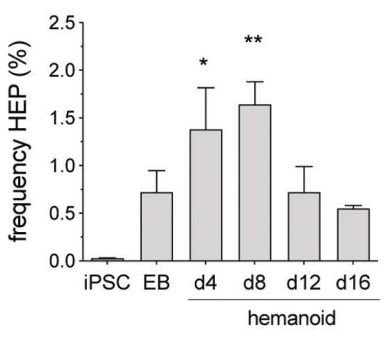

C Hemanoid dav 4
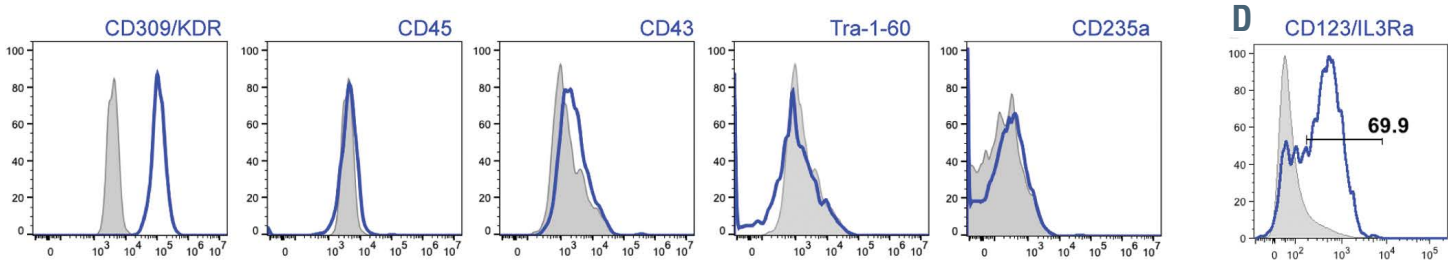

E

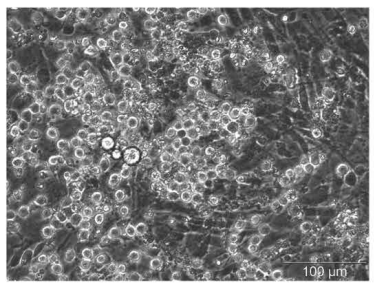

$\mathrm{F}$
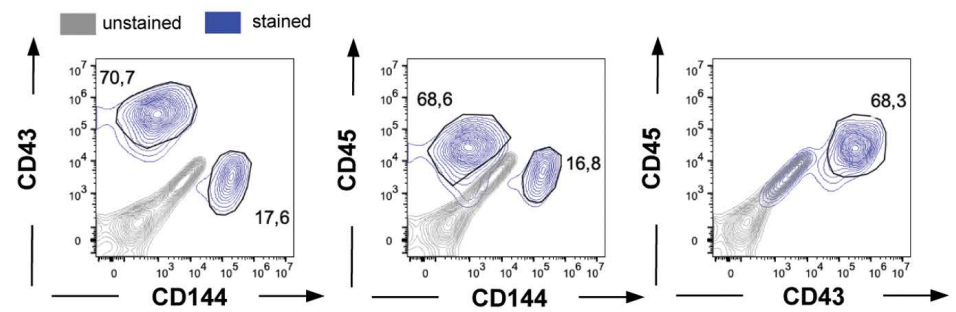

G

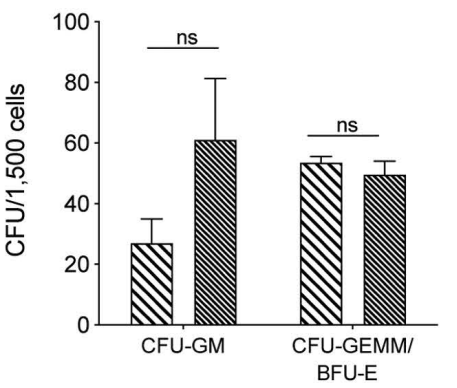

NiPSC CD34+

CB CD34+

Figure 3. Characterization of hemato-endothelial progenitors within hemanoids. (A and B) Frequency of $\mathrm{CD} 34^{+} / \mathrm{CD} 144^{+}$hemato-endothelial progenitors (HEP) at different days of hematopoietic specification. (A) Representative flow cytometry data (percentage of gated population is indicated) and (B) quantification of $n=4$, mean \pm standard error of the mean (SEM). (C) Detailed surface marker phenotype of HEP derived from hemanoids day 4 analyzed by flow cytometry (pre-gated on $\mathrm{CD}^{2} 4^{+} / \mathrm{CD} 144^{+}$cells, histogram overlay: unstained grey filled, surface marker expression blue, representative data from $\mathrm{n}=3$, approx. $1,000 \mathrm{HEP}$ were analyzed per sample). (D) Expression of the IL3Ra/CD123 on CD34 ${ }^{+}$CD144 $4^{+} \mathrm{HEP}$ (histogram overlay: unstained grey filled, surface marker expression blue, representative data from $n=3$, approx. 1,000 HEP were analyzed per sample, percentage of gated population is indicated). (E) Morphology of HEP after 1 week in endothelial to hematopoietic transition (EHT) culture (scale bars: $100 \mu \mathrm{m}$ and $200 \mu \mathrm{m}$, respectively). (F) Flow cytometry analysis of CD144, CD43 and CD45 expression on CD34 cells after 1 week EHT culture (unstained: grey, surfacemarker expression: blue, representative data from n=2, approx. 10,000 CD34 cells were analyzed per sample, percentage of gated population is indicated, pre-gating on CD34 is shown in the Online Supplementary Figure S3C). (G) Left: Frequency of colony forming units (CFU) of iPSC-derived CD34 $4^{+}$cells after 1 week of EHT culture and cord blood-derived $C D 34^{+}$cells $(n=4$, two biological and two technical replicates, mean \pm SEM). ${ }^{*} P<0.05, * * P<0.01$, ns: not significant; statistical significance was assessed using (B) One-way ANOVA with Dunnetts multiple comparison test and (G) two-way ANOVA with Sidak's multiple comparisons test.) 
ation process. To this end, EB were generated from an established iPSC line (hCD34iPSC16) ${ }^{28}$ by culture on an orbital shaker for 5 days and subsequently, cultured in media (i) without cytokines, (ii) with IL-3 only and (iii) in standard conditions containing IL-3/M-CSF (Figure 1A). While the absence of any cytokines precluded the production of hematopoietic cells from hemanoids, supplementation with IL-3 alone resulted in the continuous production of predominantly (>90\%) CD $45^{+}$blood cells that could be harvested from the supernatant over several weeks, albeit at 2-3-fold lower quantities compared to the standard IL3/M-CSF condition (Figure 1B; Online Supplementary Figure $S 1 A$ ). Interestingly, cells produced by the IL-3 only hemanoids were smaller (based on their forward scatter profile/properties in flow cytometry) and showed a more immature myeloid phenotype of $\mathrm{CD}^{+} 5^{+} / \mathrm{CD} 11 \mathrm{~b}^{+} / \mathrm{CD} 14^{-} / \mathrm{CD} 163^{-}$compared to the predominantly $\mathrm{CD}_{4} 5^{+} / \mathrm{CD} 11 \mathrm{~b}^{+} / \mathrm{CD} 14^{+} / \mathrm{CD} 163^{+}$and larger monocyte/macrophages generated by the standard IL-3/M-CSF culture condition (Figure 1C-D; Online Supplementary Figure S1A). Cells harvested from IL-3 only cultures showed a higher frequency of cells with colony forming potential, as demonstrated by a significantly higher potential to form granulocyte/monocyte colonies (CFU-GM) and erythroid burst forming units (BFU-E) or granulocyte/erythrocyte/monocyte/megakaryocyte (CFUGEMM) colonies in a methyl-cellulose-based assay (Figure 1E). Of note, the few cells generated without cytokine supplementation did not give rise to any colonies in this assay. Cells from the IL-3 only hemanoids could be further matured into iPSC-derived macrophages or granulocytes in the presence of M- or G-CSF, respectively. iPSC-derived macrophages were characterized by the expression of CD14 and CD163, whereas iPSC-derived granulocytes showed expression of CD66b and CD16 upon final differentiation (Figure 1F; Online Supplementary Figure S1B). Taken together, we show that addition of IL-3 only is sufficient to induce the hematopoietic program in our hemanoid differentiation system and enables the generation of iPSC-derived myeloid progenitor cells.

\section{Hematopoietic differentiation in human induced pluripotent stem cell-derived hemanoids recapitulates embryonic hematopoietic development}

As a next step, we aimed to further characterize the cellular composition of the hemanoids and performed hematoxilin/eosin (H/E) staining on paraffin embedded sections. Whereas a high proportion of EB resembled neuroectodermal structures, after hematopoietic specification, several hemanoids also displayed glandular, vimentin-positive mesodermal and pan-cytokeratin-positive epithelial structures (Figure 2A; Online Supplementary Figure S2A). Moreover, several hemanoids of day 16 of differentiation demonstrated cystic structures with endothelial linings as well as hematopoietic cells stained positive for CD45 (Figure 2A and B). Immunofluorescent staining moreover confirmed the development of all three germlayers in the hemanoids as we detected tubulin positive cells marking neuronal networks, FOXA2 positive endoderm as well as desmin positive mesodermal/muscle cells (Online Supplementary Figure S2B).

In order to further delineate the effect of IL-3 on early hematopoietic specifications, we next investigated early key stages of human embryonic hematopoietic development in our hemanoid system. We dissociated EB and whole hemanoids cultured with IL-3 at defined differentiation stages (days 4, 8 and 12) and analyzed the expression of specific TF associated with hematopoietic specification and the emergence of early hematopoietic cell populations. We found differential regulation of key genes involved in early human hematopoietic development within the first 16 days of differentiation. The primitive streak marker MIXL1 and early mesoderm gene KDR1 were upregulated during EB formation and subsequently progressively downregulated in the hematopoietic specification phase. Transient expression of SOX17, a key TF
A

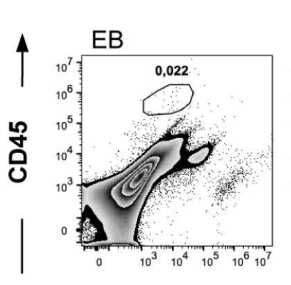

C

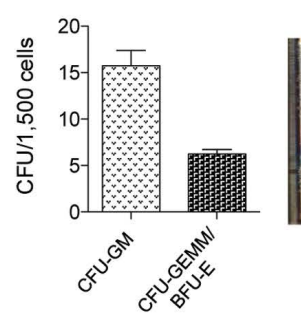

Hemanoid
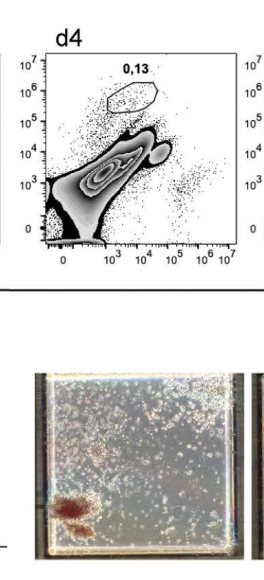

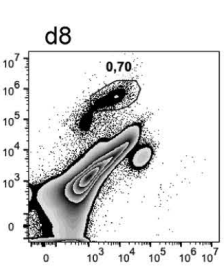

CD34
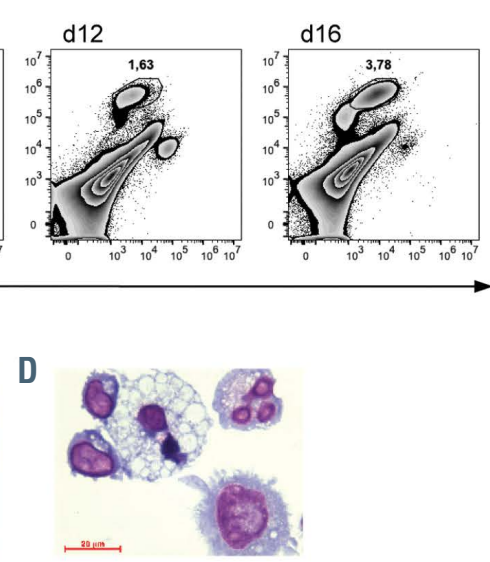

B

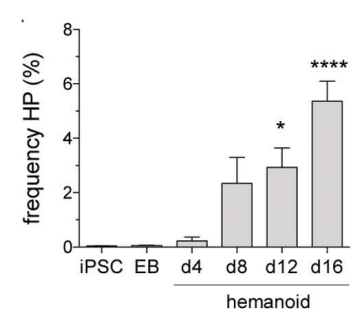

Figure 4. Characterization of hematopoietic progenitors within hemanoids. (A and B) Frequency of $\mathrm{CD} 34^{+} \mathrm{CD} 45^{+}$hematopoietic progenitors (HP) at different days of hematopoietic specification. (A) Representative flow cytometry data (percentage of gated population is indicated) and (B) quantification of $n=4$, mean \pm standard error of the mean (SEM). (C) Left: Frequency of colony-forming unites (CFU) of fluorescence-activated cell sorting-purified HP (mean \pm SEM, $n=4$, two biological and two technical replicates) and right: bright field microscopy of colony forming units (CFU) in methylcellulose. (D) Representative cytospin staining of CFU from HP derived from the clonogenic assay (scale bar: $20 \mu \mathrm{m}$ ). ${ }^{*} P<0.05, * * * * P<0.0001$; statistical significance was assessed using one-way ANOVA with the Dunnetts multiple comparison test. 
expressed in (hemogenic) endothelium was noted in early phases of differentiation (EB and hemanoid day 4 to day 8), whereas expression of the hematopoietic TF TAL1 progressively increased during the differentiation process (Figure 2C). In line with these results, we observed a strong downregulation of the pluripotency-associated surface antigen TRA-1-60 during the first days of differentiation. Moreover, expression of CD144 and CD34, both surface antigens expressed on (hemogenic) endothelium, peaked in hemanoids at day 8 of differentiation. The emergence of the first hematopoietic cells characterized by expression of CD43 and CD45 was observed around day 4 or day 8 of differentiation, respectively (Figure 2D).

A more detailed analysis of defined cell populations within the hemanoids revealed the early emergence of $\mathrm{CD} 34^{\text {high }} / \mathrm{CD} 144^{+} \mathrm{HEP}$ during EB formation and the early hematopoietic specification phase. HEP were first detected in $\mathrm{EB}(0.72 \pm 0.46 \%$, mean \pm standard deviation [SD], $\mathrm{n}=4$ ), and numbers gradually increased until hemanoid day 8 to $1.64 \pm 0.48 \%($ mean $\pm S D, n=4)$. Thereafter, a clear reduction of HEP in hemanoid day 8 to day 12 was observed (Figure $3 \mathrm{~A}$ and B). The CD $34^{\text {high }} / \mathrm{CD} 144^{+} \mathrm{HEP}$ were further characterized by high expression of CD309/KDR, absence of the hematopoietic markers CD45 and CD43 as well as the pluripotency-associated surface marker TRA-1-60. Moreover, CD235a, a marker previously associated with primitive YS hematopoiesis ${ }^{16}$ was not detectable (Figure 3C). Interestingly, $74.1 \pm 10.32 \%($ mean $\pm S D, n=3)$ of hemanoid day 8 HEP expressed the IL-3 receptor $\alpha$-chain (IL3RA, CD123) (Figure 3D). When purified from the hemanoid by fluorescence-activated cell sorting (FACS), HEP could be cultured
A
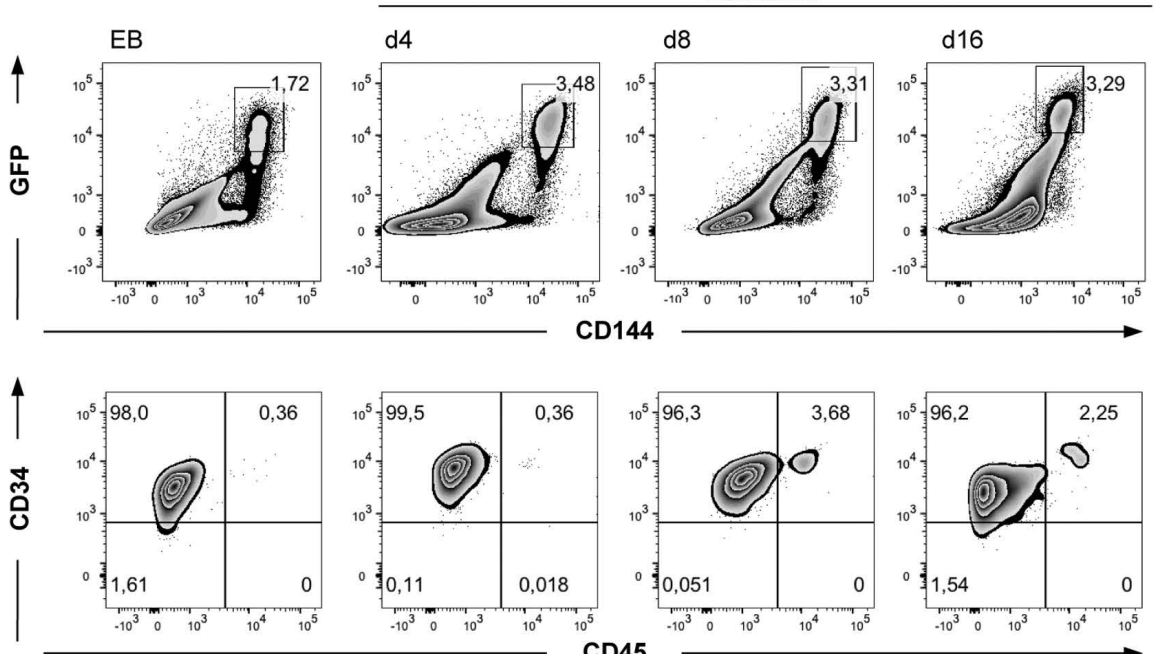

CD45

C

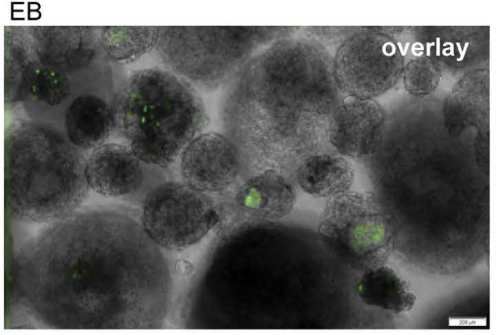

Hemanoid $_{\mathrm{d} 16}$

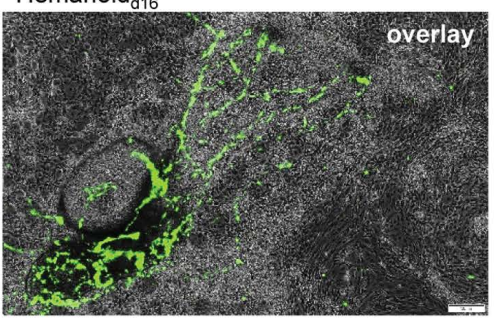

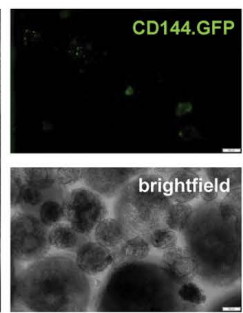
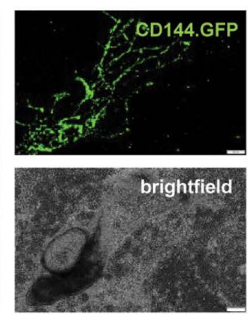

Hemanoid $_{\mathrm{d} 8}$
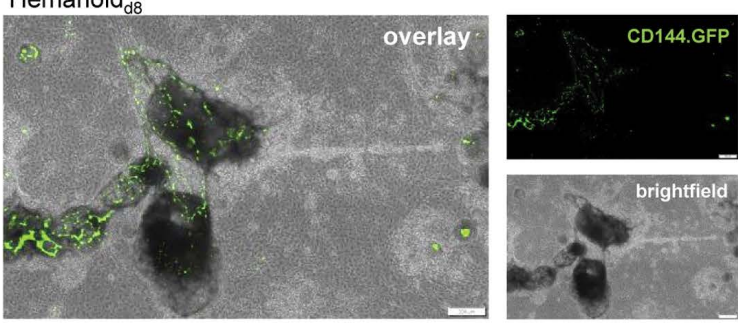

Hemanoid ${ }_{\mathrm{d} 16}$
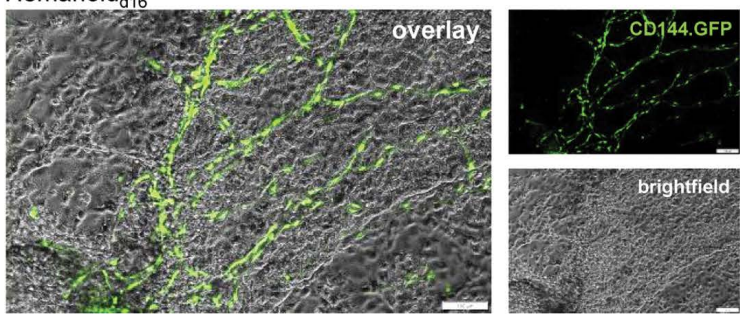

Figure 5. 3D organisation of hemato-endothelial progenitors within the hemanoids using a lentiviral reporter construct. (A) Upper panel: flow cytometry analysis correlating enhanced green fluorescent protein (eGFP) and CD144 expression in embryoid bodies (EB) and hemanoids (day 8 and day 16) generated from the transgenic human induced pluripotent stem cell line hCD34iPSC16 CBX3.CD144.GFP. Lower panel: analysis of CD34 and CD45 expression in GFP ${ }^{+} / \mathrm{CD}_{144}{ }^{+}$cells (representative data of $n=3$, percentage of gated population is indicated). (B) Expression of GFP in CD144 ${ }^{+}$CD45- (green; hemato-endothelial progenitors [HEP]), $\mathrm{CD} 144^{-} / \mathrm{CD}_{4} 5^{+}$(red, hematopoietic progenitors [HP]), and CD34- (grey) cells. Upper plot represents individual staining of CD45 and CD144 in day 16 hemanoids (pre-gated on total CD34 ${ }^{+}$cells, percentage of gated population is indicated). Lower histogram overlay shows GFP expression in HEP, HP and CD34 ${ }^{-}$cells, percentage of gated population is indicated. (C) Fluorescence microscopy of EB and developing hemanoids at day 8 and day 16 of differentiation (brightfield, CD144. GFP fluorescence and overlay, scale bar $200 \mu \mathrm{m}$ and $100 \mu \mathrm{m}$ respectively). 
A

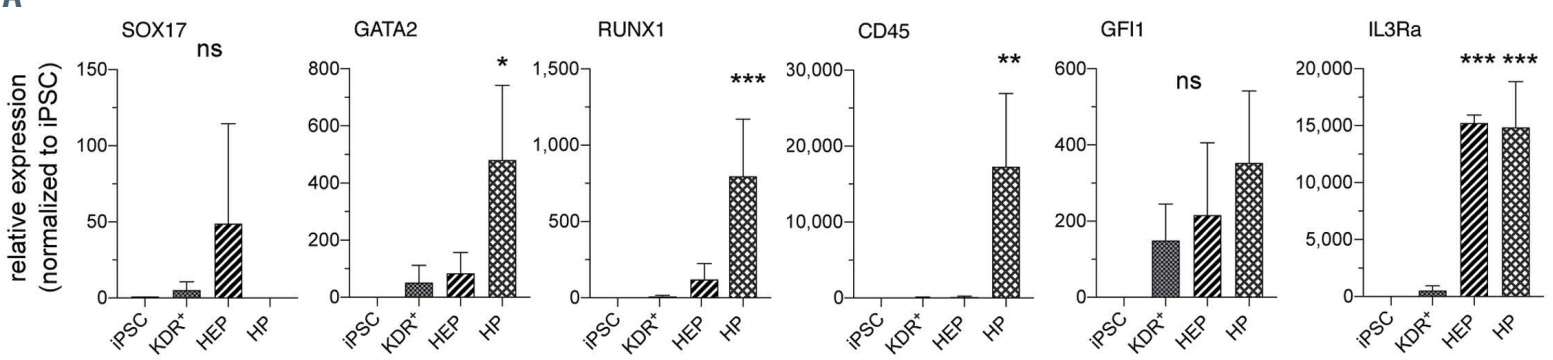

B

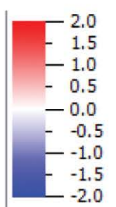

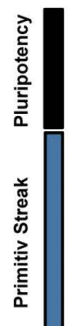

POU5F DPPA4 PODXL $\mathrm{CDH1}$

PRDM14 LEF1 SNAI2
PDGFRA MSX1

HAND1

$\mathrm{CDH11}$

WNT5A

APOE

APLNR

NOTCH4

ICAM2

LMO2

GJA4

.

ESAM

EGFL7

VASH1

HES4

NOTCH1

EFNA1

PRCP

HOXA9

CRIP2

ACVRL

SOX18

CD93

RYR3

GFI1B

NFE2

GATA1

ZBTB16

CD33

PSRY8

SYK

CSF1R

CD53

$\frac{\infty}{D}$ SPIT

क ASGR2

DHCR 7

DHCR7

ANGPT1

ITGA2B
PSRX1

GYPB

MYB

BTK

LYL1

CD69

GATA2

CX3CR

EBP

GYPE
C

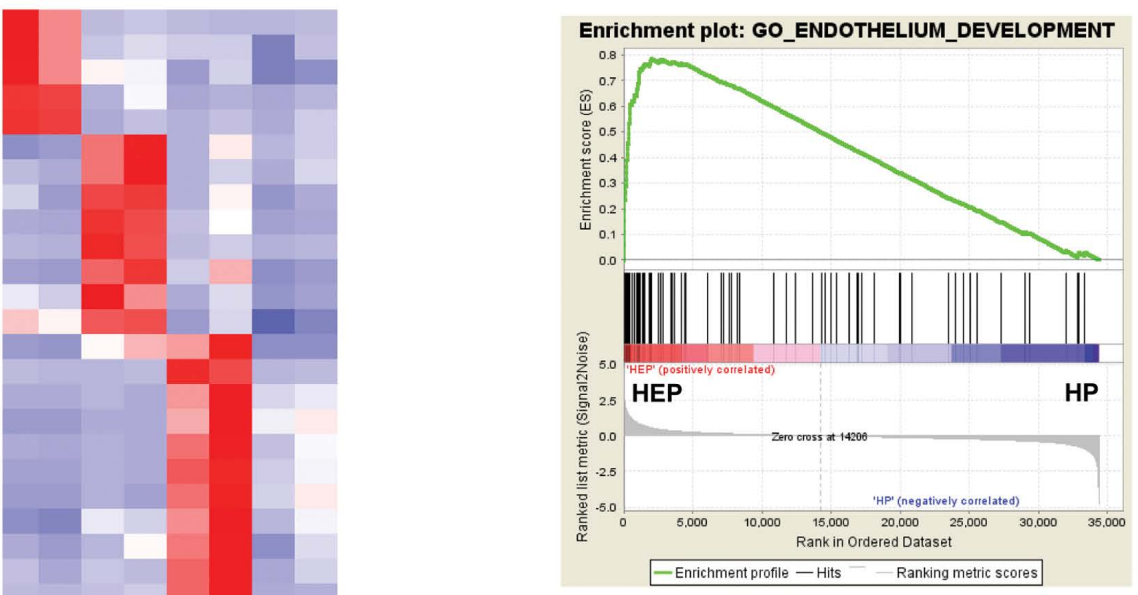

Enrichment plot:

KEGG_HEMATOPOIETIC_CELL_LINEAGE

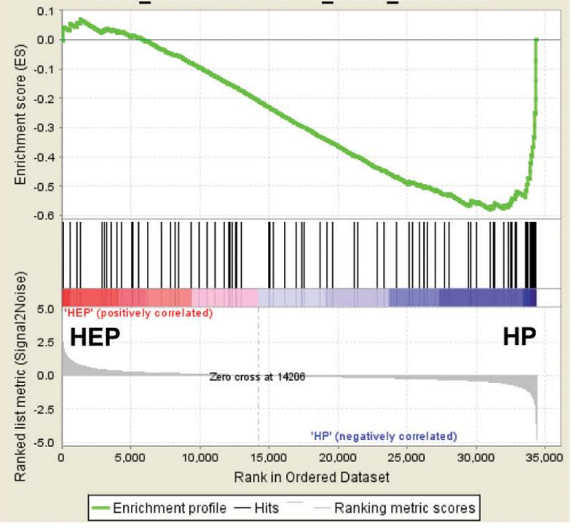

Figure 6. Whole transcriptome analysis. Fluorescence-activated cell sorting (FACS)-purified Tra-1-60+ pluripotent induced pluripotent stem cell (iPSC), Tra-1-60$/ \mathrm{KDR}+$ mesodermal progenitor cells derived from embryoid bodies (EB), CD34 ${ }^{+} / \mathrm{CD} 144^{+} / \mathrm{CD}_{4} 5^{-}$hemato-endothelial progenitor (HEP) and CD34 ${ }^{+} / \mathrm{CD} 144$ $/ \mathrm{CD} 45^{+}$hematopoietic progenitors (HP) derived from the hemanoids were analyzed by microarray analysis. (A) Expression analysis of selected genes (SOX17, GATA2, RUNX1, CD45, GFI1 and IL3RA) in FACS-purified populations by quantitative real time polymerase chain reaction (qRT-PCR) ( $\mathrm{n}=3$, mean \pm Standard error of the mean [SEM]). (B and C) Analysis of whole transcriptome data. (A) Heatmap analysis of selected GO (pluripotency: black, primitive streak: blue, blood vessel endothelium: green, hematopoiesis: red). (C) Gene set enrichment analysis of HEP and HP. Upper panel: GO Endothelium development (normalized enrichment score [NES]=2.89, $P<0.001$, false discovery rate [FDR] $<0.01$, lower panel: KEGG hematopoietic cell lineage (NES $=-1.99, P<0.001$, FDR $<0.01) . * P<0.05, * * * * P<0.0001$; statistical significance was assessed using one-way ANOVA with the Dunnetts multiple comparison test. 


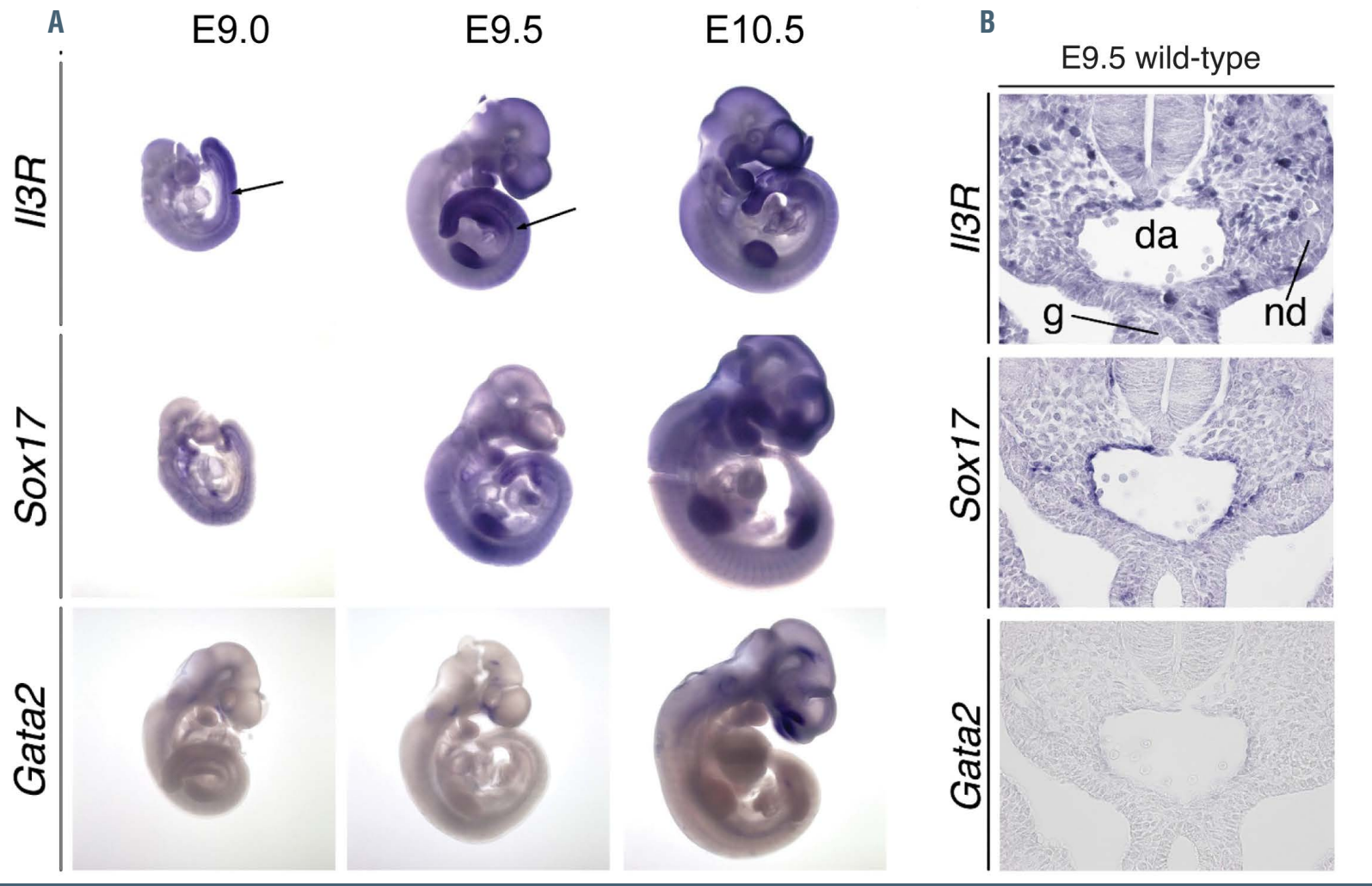

Figure 7. In situ hybridization analyses on embryonic day (E) 9.0, E9.5 and E10.5 wild-type embryos. (A) Whole mount in situ hybridization analysis of IL-3R, Sox17 and Gata2 mRNA in E9.0, E9.5 and E10.5 embryos. IL-3R was expressed in the aorta-gonad-mesonephros (AGM) region (see arrowhead), the tailbud mesenchyme, the limbs, the branchial arches and the somites from E9.0 until E10.5. Sox17 mRNA was expressed in these tissues at all analysed stages. Gata2 transcripts were found in the caudal head mesenchyme from E9.0 until E9.5 and additionally in the otic vesicle, the midbrain and the AGM region at E10.5. (B) RNA in situ hybridization analysis of transverse sections of the E9.5 embryo.

in endothelial medium for more than three passages, revealed similar endothelial morphology to human umbilical cord endothelial cells (HUVEC), and were able to form endothelial tubes in semisolid culture medium (Online Supplementary Figure $3 A$ and $B$ ). Besides this endothelial phenotype and functionality, HEP were also analyzed for their potential to undergo the EHT and to give rise to $\mathrm{CD} 34^{+} / \mathrm{CD} 45^{+}$hematopoietic progenitor cells. Coculture of FACS-purified, CD34 $/ \mathrm{CD} 144^{+} / \mathrm{CD} 45^{-} \mathrm{HEP}$ from hemanoid day 8 on OP9-DLL1 and 4 expressing stroma cells in IL-3 supplemented medium resulted in the formation of characteristic "cobblestone areas" and roundshaped suspension cells after approximately 1 week (Figure 3E). Flow cytometry confirmed the downregulation of CD144 as well as the upregulation of CD43 and CD45 in the majority of CD34+ cells and thus the acquired hematopoietic phenotype (Figure 3F; Online Supplementary Figure S3C). CD $144^{-} / \mathrm{CD} 34^{+} / \mathrm{CD} 45^{+}$ hematopoietic stem/progenitor cells from the EHT culture demonstrated high clonogenic potential in a methylcellulose assay and gave rise to different colony types with comparable frequency to cord-blood-derived CD $34^{+}$cells (Figure 3G; Online Supplementary Figure 3D). iPSC-derived hematopoietic cells isolated from the CFU represented different myeloid celltypes such as macrophages, granulocytes and erythrocytes (Online Supplementary Figure S3C). In constrast, HEP freshly purified from hemanoids and before EHT did not give rise to hematopoietic colonies when cultured in methyl-cellulose (Online Supplementary Figure S3E).
Following the emergence of HEP in the early phases of hematopoietic specification, we detected first $\mathrm{CD} 34^{\text {low }} / \mathrm{CD} 45^{+}$hematopoietic progenitors (HP) at hemanoid day 4 which increased till hemanoid day 16 to $5.36 \pm 1.46($ mean $\pm S D, n=4)$ (Figure $4 \mathrm{~A}$ and $\mathrm{B})$. HP were further characterized by the absence of CD144 and TRA-160 , low expression of CD43, and expression of the IL$3 \mathrm{R} \alpha / \mathrm{CD} 123$ (Online Supplementary Figure S3F). Functionally, HP demonstrated clonogenic potential in a methylcellulose-based assay and generated different colony types as well as several myeloid cell types (Figure $4 \mathrm{C}$ and $\mathrm{D})$.

\section{Endothelium in human induced pluripotent stem cell-derived hemanoids shows 3D organization}

Interestingly, HEP within the developing hemanoids demonstrated a highly organized 3D structure, indicated by a reporter iPSC line (hCD34iPSC) ${ }^{16}$ which was transduced with a lentiviral vector (CBX3.CD144.GFP) expressing an enhanced green fluorescent protein (eGFP) under the control of a CD144 promoter sub-fragment (modified from). ${ }^{30}$ In order to prevent epigenetic silencing, a minimal CBX3 ubiquitous chromatin opening element ${ }^{31}$ was incorporated into the lentiviral vector. Flow cytometric analysis of $\mathrm{EB}$ and hemanoids generated from the reporter line confirmed a strong and specific eGFP expression only in CD $34^{+} / \mathrm{CD} 144^{+} / \mathrm{CD} 45^{-}$cells, as well as a downregulation of eGFP expression in CD34 /CD144-/ CD45 hematopoietic progenitor cells (Figure 5A-B). When analyzing the developing hemanoids derived from the CD144-reporter 
iPSC line by fluorescence microscopy, we noted GFP expression already at the EB stage, which correlated with the emergence of few HEP detected by flow cytometry. Following differentiation, an organized and arborizing endothelial like network was formed within the hemanoids, as seen by fluorescence microscopy on different days of differentiation (Figure $5 \mathrm{C}$ ).

\section{Transcriptome analysis recapitulates defined} developmental stages

We next subjected FACS purified TRA-1-60+ pluripotent iPSC, TRA-1-60-/KDR ${ }^{+}$mesodermal progenitor cells derived from EB, CD $34^{\text {high }} / \mathrm{CD} 144^{+} / \mathrm{CD} 45^{-} \mathrm{HEP}$ and $\mathrm{CD} 34^{\text {low }} / \mathrm{CD} 144^{-} / \mathrm{CD} 45^{+} \mathrm{HP}$ derived from the hemanoids to whole transcriptome analysis. Quantitative RT-PCR on
A

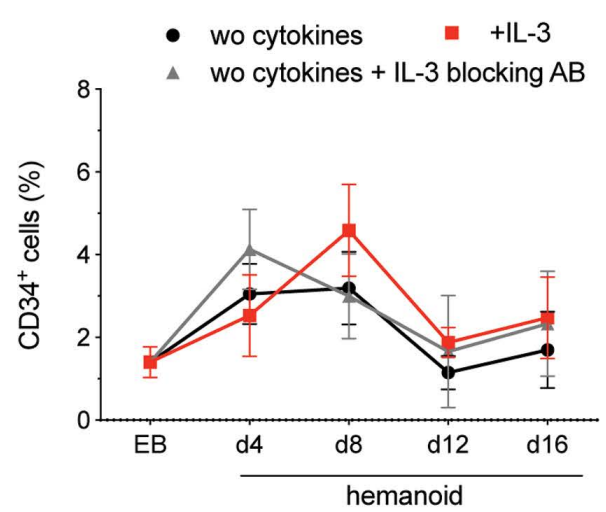

$c^{\prime}$

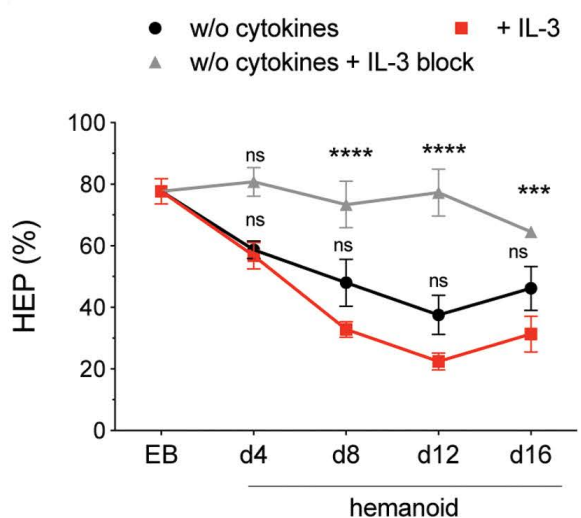

B

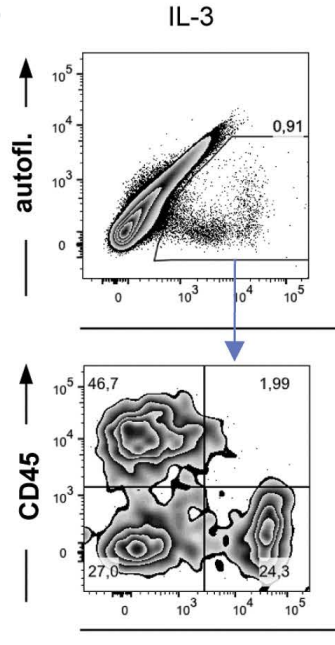

w/o cytokines
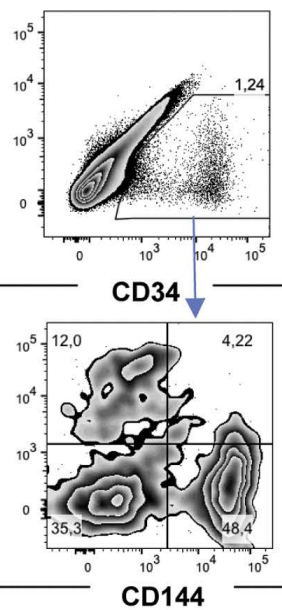

CD144

- w/o cytokines $\quad+\mathrm{IL}-3$

- w/o cytokines + IL-3 block

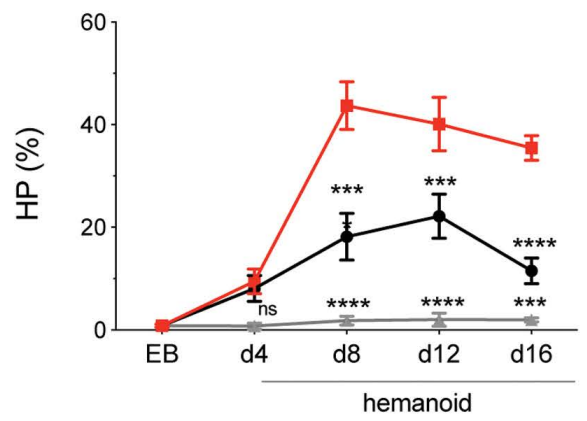

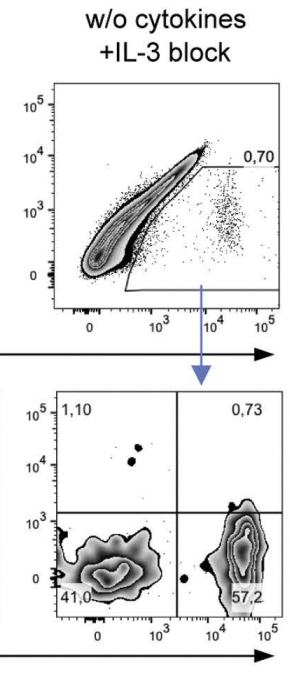

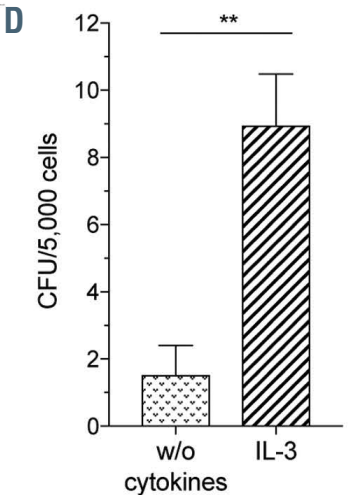

E

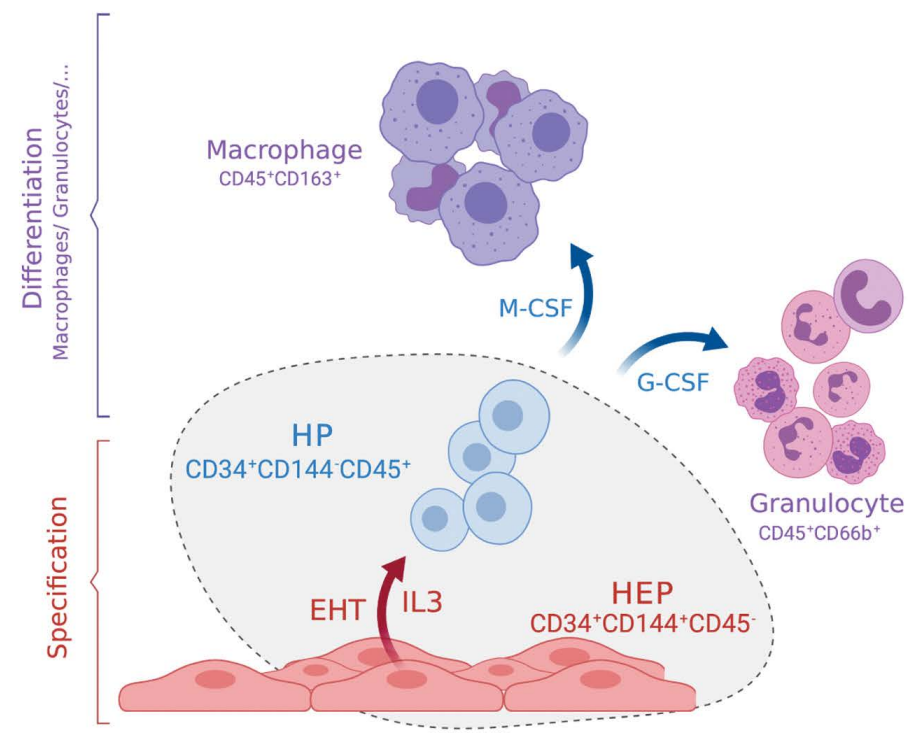

Figure 8. Influence of IL-3 on the endothelial to hematopoietic transition of hemato-endothelial progenitors. (A) Frequency of total $\mathrm{CD} 34^{+}$within hemanoids at different days cultured either without (w/o) cytokines, w/o cytokines+IL-3 blocking antibody (IL-3 block) or in the presence of IL-3 $(n=4$ 7, mean \pm standard error of the mean [SEM]). ( $B$ and $C$ ) Analysis of $\mathrm{CD}_{144^{+} / \mathrm{CD} 45-\text { hemato-endothelial progenitors }}$ (HEP) and $\mathrm{CD} 144^{-} / \mathrm{CD}_{4} 5^{+}$hematopoietic progenitors (HP) among total $\mathrm{CD}_{4} 4^{+}$cells within the hemanoids. (B) Representative flow cytometry data for hemanoid day (d) 8 and (C) quantification of $n=4-7$ over 16 days of hematopoietic specification, mean $\pm S E M$, percentage of gated population is indicated). (D) Colony forming units (CFU) in methyl-cellulose after endothelial to hematopoietic transition (EHT) culture on OP9 stromal cells in the presence or absence of IL-3 $(n=4$, two technical and two biological replicates, mean \pm SEM). (E) Schematic representation of the hemanoid model system as well as the effect of IL-3 on the hematopoietic specification of hemogenic endothelium (scheme was prepared with BioRender software) $* P<0.05, * * P<0.01, * * * P<0.001$ $* * * * P<0.0001$, ns: not significant; statistical significance was assessed using two-way ANOVA with Tukey's multiple comparison test. 
sorted populations confirmed the high expression of SOX17 in HEP, whereas the hematopoietic TF GATA2 and RUNX1 as well as the protein tyrosine phosphatase CD45 were particularly expressed in HP. We furthermore evaluated the expression of the TF GFI1, which has been shown to play a crucial role in the intra-embryonic EHT. ${ }^{32}$ In the hemanoids, GFI1 expression was first observed in $\mathrm{KDR}^{+}$mesoderm and increased during further differentiation stages from HEP to HP. Quantitative PCR as well as whole transcriptome analysis confirmed increased expression of the IL3Ra already at the HEP stage (Figure $6 \mathrm{~A}$ ). In order to verify this early expression of the IL-3 receptor, we performed RNA in situ hybridization of E9.5 murine embryos, a developmental stage where Sox $17^{+}$ hemogenic endothelium has already developed in the AGM, but no hematopoietic cells characterized by expression of Gata2 are yet detectable. Also in this in vivo scenario we observed partial co-expression of the IL3ra and Sox17 in cells lining the dorsal aorta before the EHT, although IL3ra appeared to be more widely expressed in the AGM domain (Figure 7).

Expression analysis of stage-specific gene ontologies in whole transcriptome data demonstrated characteristic expression profiles of all defined cell populations. Transcripts associated with pluripotency (black) such as NANOG, DNMT3B, and POUSF1 were highly expressed in iPSC and rapidly downregulated upon induction of hematopoietic differentiation. In contrast, genes associated with primitive streak and mesoderm (dark blue) formation such as PDGFRA, HAND1 or CDH11 were highly enriched in the Tra-1-60-/KDR ${ }^{+}$early mesoderm population. Emerging HEP showed characteristic expression of transcripts associated with blood vessel endothelium (green) such as genes involved in transforming growth factor (TGF) (e.g., ACVRL1) and Notch (NOTCH1, DLL4) signaling. When analyzing gene ontologies associated with hematopoiesis (red) we found the important regulator of EHT HOXA9 highly expressed in our HEP, further confirming their hematopoietic potential. Classical genes involved in hematopoiesis such as GFI1B, GATA2, GATA1, EVI2B, SPI1 or ANGPT1 were highly expressed in HP (Figure $6 \mathrm{~B}$ ). Gene set enrichment analysis confirmed the endothelial phenotype of HEP as well as the upregulation of genes specific for HSC in HP (Figure 6C). Further analysis of HEP revealed an upregulation of genes associated with an arterial endothelium such as DLL4, EFNB2 and NOTCH1, whereas markers of venous endothelial fate did not reveal gene upregulation, except from EPHB4 (Online Supplementary Figure S4A and B, respectively). Next, we analyzed the signaling pathways important for the development of iPSC towards HP. Signaling pathways at the defined differentiation stages revealed the glycine, serine and threonine metabolism pathways as well as hedgehog and WNT signaling pathways were highly enriched in the $\mathrm{KDR}^{+}$early mesoderm population sorted from EB (EnrichR, KEGG2018). In contrast, developing HEP showed highest enrichment scores for hematopoietic stem cell differentiation, angiogenesis, and dorsal aorta development (EnrichR, GO Biological Processes), whereas in HP, especially genes associated with IL5, IL-3 or B-cell receptor signaling were upregulated when compared to HEP (EnrichR, WikiPathways, Online Supplementary Figure S4C).

Taken together, our hemanoid system recapitulates early human hematopoietic development and shows the temporally regulated emergence of $\mathrm{KDR}^{+} / \mathrm{CD} 34^{\text {high }}$ / CD $144^{+} / \mathrm{CD}_{43}{ }^{-} \mathrm{CD} 45^{-}$HEP with endothelial and hematopoietic potential and a characteristic transcriptional fingerprint. Also the emergence of clonogenic $\mathrm{CD} 34^{\text {low }} / \mathrm{CD} 144^{-} / \mathrm{CD} 43^{+} \mathrm{CD} 45^{+} \mathrm{HP}$ expressing important TF characteristic for hematopoietic stem and progenitor cells was observed at later stages of differentiation.

\section{Influence of IL-3 on the early hematopoietic} specification of human induced pluripotent stem cells

After defining the spatiotemporal emergence of human HEP and HP in our hemanoid system, we next evaluated the effect of IL-3 on the emergence of early HP. We dissociated whole hemanoids cultured in different cytokine conditions at specific days of differentiation and analyzed the frequency of HEP and HP populations. No significant difference in the overall frequency of emerging CD34 cells irrespective of the addition of IL-3 could be observed. Similarly, cultures treated with an anti-IL-3 antibody to block any effect by autocrine or paracrine secreted IL-3 within the hemanoids, revealed a similar frequency of total $\mathrm{CD} 4^{+}$cells (Figure 8A-B). However, when further analyzing the fate of $\mathrm{CD} 34^{+}$cells within the hemanoids by scoring CD144 (HEP) or CD45 (HP) expression, we noted strong differences in cultures treated with IL-3, compared to cultures in which IL-3 was omitted (w/o) or blocked by the addition of an IL-3 blocking antibody (w/o + IL-3 block). In IL-3 treated cultures or cultures w/o cytokines analyzed at day 8 of differentiation $\mathrm{CD}_{3} 4^{+}$contained CD34 $4^{\text {high }} / \mathrm{CD} 144^{+}$and $\mathrm{CD} 34^{\text {low }} / \mathrm{CD} 45^{+}$positive subpopulations (Online Supplementary Figure S5A). In contrast, nearly all $\mathrm{CD} 34^{+}$cells remained $\mathrm{CD} 144^{+}$and were unable to undergo the EHT process in cultures treated with an IL3 blocking antibody (Figure 8B). Moreover, CD34 ${ }^{+}$cells in hemanoids supplemented with IL-3 showed a decline in $\mathrm{CD} 34^{\text {high }} / \mathrm{CD} 144^{+} / \mathrm{CD} 45^{-} \mathrm{HEP}$ cells and an increasing proportion of CD45 expressing cells over time, suggesting successful EHT and the progression towards a CD34 low $/ \mathrm{CD} 144^{-} / \mathrm{CD} 45^{+} \mathrm{HP}$ phenotype (Figure 8C). Interestingly, also without the exogenous addition of IL-3 we observed the emergence of $18.18 \pm 11,16 \%$ (mean \pm SD, $\mathrm{n}=6) \mathrm{CD} 34^{\mathrm{low}} / \mathrm{CD} 144^{-} / \mathrm{CD}_{4} 5^{+} \mathrm{HP}$ in hemanoid day 8 , an effect that could be completely blocked upon addition of an anti-IL-3 antibody to the differentiation cultures $(1.85 \pm 1,90 \% \mathrm{HP}$, mean $\pm \mathrm{SD}, \mathrm{n}=5)$. This data suggests local production of IL-3 by the hemanoid complex, which is sufficient to induce the EHT of some HEP (Figure 8C). In order to further specify at which stage the EHT is arrested upon IL-3 inhibition, we analyzed the expression of CD43, one of the earliest surface markers indicating the hematopoietic fate of human embryonic endothelium. ${ }^{33}$ Also the emergence of $\mathrm{CD} 34^{+} / \mathrm{CD} 144^{-} / \mathrm{CD} 43^{+}$was strongly reduced in cultures without addition of IL-3 and this effect was even more pronounced in cultures where autocrine/paracrine IL-3 signaling was blocked (Online Supplementary Figure S5B). In order to further assess the important role of IL-3 in the progression of HEP to HP, we subjected FACS-purified HEP from the hemanoid system to the OP-9 based in vitro EHT culture assay. Similar to our 3D hemanoid culture, we observed significantly more ( $>5$ fold) CFU when IL-3 was added to the EHT culture medium compared to culture conditions without IL-3 (Figure 8D).

Of note, the stimulating effect of IL-3 on the EHT could not be recapitulated by the addition of Oncostatin $M$ or 
stem cell factor (SCF), both described as regulators of early hematopoietic development. While we did not see a change in the frequency of emerging HEP in the hemanoids, we observed a significant increase of HP only in cultures treated with IL-3, but not upon addition of Oncostatin M (Online Supplementary Figure S5C). Similarly, also the addition of SCF, independent of a simultaneous block of endogenous IL-3 signaling, did not lead to an increase in the frequency of HP compared to cultures without cytokine supplementation. Also, the combined supplementation of differentiation cultures with IL-3 and SCF did not lead to an increase in HP compared to cultures with only IL-3 supplementation (Online Supplementary Figure S5D).

Given the heterogeneity between different iPSC lines, we confirmed our key findings in a second iPSC line, which was derived from fetal fibroblasts (F134iPSC). ${ }^{34}$ Similar to our previous finding, the addition of only IL-3 resulted in the efficient production of hematopoietic cells from the hemanoids (Online Supplementary Figure S6A). In addition, the transition of HEP towards HP was again significantly improved by the addition of IL-3, highlighting the regulatory role of IL-3 signaling in our hemanoid system (Online Supplementary Figure S6B to D). Of note, in this iPSC line, we did not observe an effect of the IL-3-blocking antibody, indicating that endogenous expression of IL3 is not a uniform feature of the hemanoids and might differ between different iPSC lines, regardless of their hematopoietic differentiation potential upon cytokine supplementation.

In summary, we here highlight the potential use of a hemanoid-based iPSC model to study human hematopoietic development and demonstrate the important role for IL-3 in early human hematopoiesis by supporting the EHT of hemato-endothelial progenitor cells (Figure 8E).

\section{Discussion}

Although well studied in adult hematopoiesis, the role of cytokines in general and IL-3 in particular remains largely unexplored during human hematopoietic development. Utilizing an iPSC-based differentiation system, which recapitulates key stages of embryonic hematopoiesis, we here demonstrate an important role for IL-3 in the endothelial to EHT of $\mathrm{KDR}^{+} / \mathrm{CD} 144^{+} / \mathrm{CD} 34^{\text {high }} / \mathrm{CD}^{2} 3^{-} /$ CD45- HEP.

We used a hematopoietic differentiation protocol, which maintains the $3 \mathrm{D}$ organization of iPSC-derived cells developing within an EB and introduced "hemanoids" as a model system to study early human hematopoietic specification. Notably, this system does not rely on excessive cytokine priming and might follow a more physiological differentiation process, as only low frequencies of $\mathrm{CD}_{3} 4^{+}$cells are detected. HEP developing within the hemanoids started to form endothelial tube-like structures already during the first days of differentiation and formed an organized/arborizing vasculature-like network within 8 to 16 days. A similar effect was recently observed by using a SOX17/RUNX1C transgenic hESC reporter-line. ${ }^{35}$ The authors demonstrated the development of $\mathrm{HOXA}^{+}$hemogenic vasculature which resembles the AGM within an EB-based protocol. Interestingly, a simultaneous modulation of activin and WNT signaling by SB/CHIR during mesoderm patterning was necessary to induce the development of an organized vasculature. In contrast, in our hemanoid system we observed the formation of vascular structures without further exogenous modification of these two pathways. Moreover, transcriptome profiling of developing HEP confirmed the upregulation of genes associated with Notch signaling, an important regulator of EHT in hemogenic endothelium and indispensable for lympho-myeloid hematopoiesis and HSC-specification. ${ }^{36,37}$ Further pathway analysis revealed high scores for dorsal aorta development/regulation in HEP compared to HP, indicating the hemogenic capacity of this cell population. Likewise, analysis of specific genes involved in arterial and venous development indicate an arterial fate of the developing endothelium, an important prerequisite for the development of definitive hemogenic endothelium. ${ }^{38,39}$

In fact, the idea of maintaining the $3 \mathrm{D}$ organization of iPSC-derived cells during the differentiation process has been applied in a variety of different tissues such as kidney, liver, brain or gut in organoid-based differentiation systems. ${ }^{19}$ Those organoids are characterized by the selforganization of cells within a 3D complex, have been shown to recapitulate human organ development remarkably similar to in vivo development and especially support the often problematic final maturation of iPSC-derived progeny as e.g., demonstrated for iPSC-derived hepatocytes. ${ }^{40,41}$ Thus, it is likely that the recapitulation of the spatial organization and structures formed within the AGM might also support the maturation and definitive hematopoietic development of human iPSC, which remains hampered without genetic modification. However, it is worth mentioning that $\mathrm{Ng}$ and colleagues did not achieve long-term engraftment with the iPSCderived cells from $3 \mathrm{D}$ cultures either, ${ }^{35}$ suggesting that important cues are still missing. Considering the onset of blood flow as an important instructive developmental signal in the early embryo, ${ }^{42,43}$ maybe this or other important determinants have to be considered in more detail and implemented into the organoid-like differentiation systems.

After we confirmed the suitability of our hemanoidbased differentiation protocol to recapitulate human hematopoietic development, we aimed to investigate the role of IL-3 on the hematopoietic specification of human iPSC. Although intensively studied during adult hematopoiesis, the role of IL-3 in early human hematopoietic development remains as of yet elusive. We demonstrate by flow cytometry and gene expression analysis, that HEP already express IL3RA before the EHT, indicating a responsiveness to IL-3 signaling. We also confirmed the physiological relevance of this finding in murine embryos and demonstrate the expression of the IL-3R in SOX $17^{+}$, GATA2 ${ }^{-}$E9.5 AGM cells. This observation is in line with previous reports, showing expression of IL-3R at very early stages of murine embryogenesis on E10.5 and E7.5 hemangioblast cells. ${ }^{12}$

Withdrawal of IL-3 or inhibition of IL-3 signaling arrested the hematopoietic specification at the hemogenic endothelium stage. This observation concurs with previous reports studying the role of IL-3 in early murine hematopoietic development. As an example, Robin and colleagues proved an IL-3 dependent amplification effect on the HSC pool in midgestation mouse embryos and showed, that IL-3 functions either as a proliferation and/or survival factor for the earliest HSC in the embryo. 
Likewise, IL-3 promotes HSC activity in E10 AGM and YS (at a time when normally no HSC are yet detected), suggesting an even earlier role of IL-3 during murine hematopoietic development. Furthermore, IL-3 has been demonstrated to elevate numbers as well as intrinsic hematopoietic commitment of the hemangioblast cells by inducing the downregulation of Flk-1 and upregulation of hematopoietic genes in single cell suspension derived from E7.5 embryos. ${ }^{12}$ These results are in line with our observations, showing that also in the human system, IL-3 can support the EHT and the emergence of $\mathrm{CD} 144^{-} / \mathrm{CD} 34^{\text {low }} / \mathrm{CD} 3^{+} / \mathrm{CD} 45^{+} \mathrm{HP}$ with clonogenic potential.

Previous studies revealed IL-3 expressing cells within the lumen of the aorta in E11 murine embryos, indicating a physiological role of paracrine IL-3 in HSC regulation. In our hemanoid system, we also observed a more pronounced differentiation arrest when an IL-3 blocking antibody was applied, suggesting the endogenous production of IL-3 within our hemanoids. This observation favors the concept, that within the hemanoids specific developmental stages of embryonic development are recapitulated and also a specific microenvironment supporting the early hematopoiesis develops. However, endogenous IL-3 production was only observed in one of the employed iPSC lines, indicating that there is some heterogeneity in the differentiation. Here, further studies are needed to evaluate the differences of endogenous IL-3 production and to determine the source of IL-3 within the hemanoid microenvironment.

Despite the similarities of our hemanoid system to the murine in vivo scenario, a contradictory role of IL-3 has been reported. Rybtsov et al. propose that IL-3 only acts on E11 Type II pre-HSC (CD144+CD41 low $\left.C D 43^{+} \mathrm{D} 45^{-}\right)$, whereas SCF is the driving factor to induce engraftment potential and the emergence of definitive HSC from murine E9 pro-HSC (CD144+CD41 $\left.{ }^{+} \mathrm{RUNX1} 1^{+} \mathrm{CD} 43^{-} \mathrm{D} 45^{-}\right)$. In our human model, however, we did not observe an effect of SCF on the EHT of HEP $\left(\mathrm{CD} 144^{+} \mathrm{CD} 34^{+} \mathrm{CD} 43^{-} \mathrm{CD} 45^{-}\right)$and subsequent emergence of either $\mathrm{CD} 43^{+}$or $\mathrm{CD} 45^{+}$cells, which only emerge when IL-3 signaling is active. However, the different subtypes are not that specifically defined in the human system and more studies are needed to shed further light onto the exact mechanism of human hematopoietic development.
In fact, our model would be of high value to study the effect of other cytokines known to promote the generation of HSC in the adult system. Given the minimal cytokine administration schedule, it would be of great interest to study the effect of e.g., FLT3-L,TPO or other cytokines to improve robust HSC production in the future.

Our hemanoid system provides proof-of-evidence, that the spatial organization of developing cells in vitro has an effect on early human hematopoietic specification and that organoid-like structures generated from hPSC may be used to unravel new determinants in the generation of de novo HSC in the near future.

\section{Disclosures}

$M A, H K$, and NL are authors od a pending patent application: "Stem-cell derived myeloid cells, generation and use thereof, EP 17169 454.0". All authors declare no conflict of interest.

\section{Contributions}

MA designed the study, performed research, analyzed and interpreted data, and wrote the manuscript; NL designed the study, interpreted data and wrote the manuscript; $K H, H K, P K$, $A C W, D H, M A, M P K, S M$ and AHNN performed research and analyzed data; $A K, M M, D J$ and TT designed research, all authors reviewed the manuscript.

\section{Acknowledgments}

The authors thank Theresa Buchegger and Doreen Lüttge for excellent technical support. We are also grateful to Prof. Dr. T. Moritz for critical comments and support. We are grateful to M. Ballmaier (Cell Sorting Facility, Hannover Medical School). We moreover thank O. Papp and Prof. Dr. T Cantz for providing the F134iPSC.

\section{Funding}

This work was supported by grants from Hannover Medical School (Hochschulinterne Leistungsförderung [HiLF] to MA), the Joachim Herz Stiftung (Add-on Fellowship to MA), the Deutsche Forschungsgemeinschaft (Cluster of Excellence REBIRTH; Exc 62/3 to NL as well as DFG LA 3680/2-1), the European Union (ERC XHaLe to DJ) and the Else Kröner-Fresenius-Stiftung (EKFS; 2015_A92 to NL and EKFS; 2016_A146 to MA). This project has received funding from the European Research Council (ERC) under the European Union's Horizon 2020 research and innovation program (grant agreement no. 852178)

\section{References}

1. Dzierzak E, Speck NA. Of lineage and legacy: the development of mammalian hematopoietic stem cells. Nat Immunol. 2008;9(2):129-136.

2. Kauts ML, Vink CS, Dzierzak E. Hematopoietic (stem) cell development how divergent are the roads taken? FEBS Lett. 2016;590(22):3975-3986.

3. Bertrand JY, Chi NC, Santoso B, Teng S, Stainier DY, Traver D. Haematopoietic stem cells derive directly from aortic endothelium during development. Nature. 2010; 464(7285):108-111.

4. Kissa K, Herbomel P. Blood stem cells emerge from aortic endothelium by a novel type of cell transition. Nature. 2010; 464(7285):112-115.

5. Zovein AC, Hofmann JJ, Lynch M, et al. Fate tracing reveals the endothelial origin of hematopoietic stem cells. Cell Stem Cell. 2008;3(6):625-636.

6. Slukvin II. Generating human hematopoietic stem cells in vitro -exploring endothelial to hematopoietic transition as a portal for stemness acquisition. FEBS Lett. 2016; 590(22):4126-4143

7. Zhu J, Emerson SG. Hematopoietic cytokines, transcription factors and lineage commitment. Oncogene. 2002;21(21):32953313

8. Ihle JN, Keller J, Oroszlan S, et al. Biologic properties of homogeneous interleukin 3. I. Demonstration of WEHI-3 growth factor activity, mast cell growth factor activity, $p$ cell-stimulating factor activity, colony-stimulating factor activity, and histamine-producing cell-stimulating factor activity. J Immunol. 1983;131(1):282-287.

9. Lantz CS, Boesiger J, Song CH, et al. Role for interleukin-3 in mast-cell and basophil development and in immunity to parasites. Nature. 1998;392(6671):90-93

10. Nishinakamura R, Nakayama $N$, Hirabayashi Y, et al. Mice deficient for the IL-3/GM-CSF/IL-5 beta c receptor exhibit lung pathology and impaired immune response, while beta IL3 receptor-deficient mice are normal. Immunity. 1995;2(3):211222.

11. Robin C, Ottersbach K, Durand C, et al. An unexpected role for IL-3 in the embryonic development of hematopoietic stem cells. Dev Cell. 2006;11(2):171-180.

12. He WY, Lan Y, Yao HY, et al. Interleukin-3 promotes hemangioblast development in mouse aorta-gonad-mesonephros region. Haematologica. 2010;95(6):875-883.

13. Ackermann M, Liebhaber S, Klusmann JH, Lachmann N. Lost in translation: pluripotent stem cell-derived hematopoiesis. EMBO 
Mol Med. 2015·7(11):1388-1402.

14. Slukvin, II. Deciphering the hierarchy of angiohematopoietic progenitors from human pluripotent stem cells. Cell Cycle. 2013;12(5):720-727.

15. Julien E, El Omar R, Tavian M. Origin of the hematopoietic system in the human embryo. FEBS Lett. 2016;590(22):3987-4001.

16. Garcia-Alegria E, Menegatti S, Fadlullah MZH, Menendez P, Lacaud G, Kouskoff V. Early human hemogenic endothelium generates primitive and definitive hematopoiesis in vitro. Stem Cell Reports. 2018;11(5):10611074.

17. Sturgeon CM, Ditadi A, Awong G, Kennedy M, Keller G. Wnt signaling controls the specification of definitive and primitive hematopoiesis from human pluripotent stem cells. Nat Biotechnol. 2014;32(6):554-561.

18. Richard C, Drevon C, Canto PY, et al. Endothelio-mesenchymal interaction controls runx1 expression and modulates the notch pathway to initiate aortic hematopoiesis. Dev Cell. 2013;24(6):600-611.

19. Lancaster MA, Knoblich JA. Organogenesis in a dish: modeling development and disease using organoid technologies. Science. 2014;345(6194):1247125.

20. Ackermann M, Lachmann N, Hartung S, et al. Promoter and lineage independent antisilencing activity of the A2 ubiquitous chromatin opening element for optimized human pluripotent stem cell-based gene therapy. Biomaterials. 2014;35(5):1531-1542.

21. Lachmann N, Ackermann M, Frenzel E, et al. Large-scale hematopoietic differentiation of human induced pluripotent stem cells provides granulocytes or macrophages for cell replacement therapies. Stem Cell Reports. 2015;4(2):282-296.

22. Ng ES, Davis R, Stanley EG, Elefanty AG. A protocol describing the use of a recombinant protein-based, animal product-free medium (APEL) for human embryonic stem cell differentiation as spin embryoid bodies. Nat Protoc. 2008;3(5):768-776.

23. Ackermann M, Kempf H, Hetzel M, et al. Bioreactor-based mass production of human iPSC-derived macrophages enables immunotherapies against bacterial airway infections. Nat Commun. 2018;9(1):5088.
24. Bernecker C, Ackermann M, Lachmann N et al. Enhanced ex vivo generation of erythroid cells from human induced pluripotent stem cells in a simplified cell culture system with low cytokine support. Stem Cells Dev. 2019;28(23):1540-1551.

25. Dreyer AK, Hoffmann D, Lachmann N, et al. TALEN-mediated functional correction of $\mathrm{X}$-linked chronic granulomatous disease in patient-derived induced pluripotent stem cells. Biomaterials. 2015;69:191-200.

26. Happle C, Lachmann N, Ackermann M, et al. Pulmonary transplantation of human induced pluripotent stem cell-derived macrophages ameliorates pulmonary alveoar proteinosis. Am J Respir Crit Care Med. 2018;198(3):350-360.

27. Kuhn A, Ackermann M, Mussolino C Cathomen T, Lachmann N, Moritz T. TALEN-mediated functional correction of human iPSC-derived macrophages in context of hereditary pulmonary alveolar proteinosis. Sci Rep. 2017;7(1):15195

28. Lachmann N, Happle C, Ackermann M, et al. Gene correction of human induced pluripotent stem cells repairs the cellular phenotype in pulmonary alveolar proteinosis. Am J Respir Crit Care Med. 2015 189(2):167-182

29. Neehus AL, Lam J, Haake K, et al. Impaired IFN $\gamma$-signaling and mycobacterial clearance in IFNy-R1-deficient human iPSC-derived macrophages. Stem Cell Reports. 2018; 10(1):7-16.

30. Prandini MH, Dreher I, Bouillot S, Benkerri S, Moll T, Huber P. The human VE-cadherin promoter is subjected to organ-specific regulation and is activated in tumour angiogenesis. Oncogene. 2005;24(18):2992-3001.

31. Muller-Kuller U, Ackermann M, Kolodziej S et al. A minimal ubiquitous chromatin opening element (UCOE) effectively prevents silencing of juxtaposed heterologous promoters by epigenetic remodeling in multipotent and pluripotent stem cells. Nucleic Acids Res. 2015;43(3):1577-1592

32. Thambyrajah R, Mazan M, Patel R, et al. GFI1 proteins orchestrate the emergence of haematopoietic stem cells through recruitment of LSD1. Nat Cell Biol. 2016;18(1):2132
33. Vodyanik MA, Thomson JA, Slukvin, II Leukosialin (CD43) defines hematopoietic progenitors in human embryonic stem cell differentiation cultures. Blood. 2006; 108(6):2095-2105

34. Sgodda M, Mobus S, Hoepfner J, et al Improved hepatic differentiation strategies for human induced pluripotent stem cells. Curr Mol Med. 2013;13(5):842-855

35. Ng ES, Azzola L, Bruveris FF, et al. Differentiation of human embryonic stem cells to $\mathrm{HOXA}(+)$ hemogenic vasculature that resembles the aorta-gonadmesonephros. Nat Biotechnol. 2016 34(11):1168-1179.

36. Ditadi A, Sturgeon CM, Tober J, et al Human definitive haemogenic endothelium and arterial vascular endothelium represen distinct lineages. Nat Cell Biol. 2015; 17(5):580-591

37. Uenishi GI, Jung HS, Kumar A, et al $\mathrm{NOTCH}$ signaling specifies arterial-type definitive hemogenic endothelium from human pluripotent stem cells. Nat Commun. 2018;9(1):1828.

38. Park MA, Kumar A, Jung HS, et al Activation of the arterial program drives development of definitive hemogenic endothelium with lymphoid potential. Cell Rep. 2018;23(8):2467-2481.

39. Slukvin II, Uenishi GI. Arterial identity of hemogenic endothelium: a key to unlock definitive hematopoietic commitment in hPSC cultures. Exp Hematol. 2019;71:3-12.

40. Asai A, Aihara E, Watson C, et al. Paracrine signals regulate human liver organoid maturation from induced pluripotent stem cells. Development. 2017;144(6):1056-1064

41. Choi YJ, Kim H, Kim JW, Yoon S, Park HJ. Hepatic esterase activity is increased in hepatocyte-like cells derived from human embryonic stem cells using a 3D culture system. Biotechnol Lett. 2018:40(5):755-763.

42. Diaz MF, Li N, Lee HJ, et al. Biomechanica forces promote blood development through prostaglandin E2 and the cAMP-PKA signaling axis. J Exp Med. 2015;212(5):665-680.

43. North TE, Goessling W, Peeters M, et al Hematopoietic stem cell development is dependent on blood flow. Cell. 2009, 137(4):736-748. 